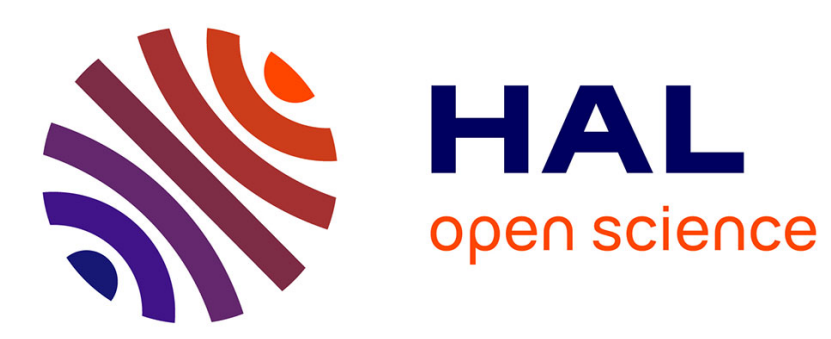

\title{
Les néonaticides dans la presse française : 357 décès suspects de 1993 à 2012 \\ Laurence Simmat-Durand, Natacha Vellut
}

\section{To cite this version:}

Laurence Simmat-Durand, Natacha Vellut. Les néonaticides dans la presse française: 357 décès suspects de 1993 à 2012. Déviance et Société, 2017. hal-01984845

\section{HAL Id: hal-01984845 \\ https://hal.science/hal-01984845}

Submitted on 17 Jan 2019

HAL is a multi-disciplinary open access archive for the deposit and dissemination of scientific research documents, whether they are published or not. The documents may come from teaching and research institutions in France or abroad, or from public or private research centers.
L'archive ouverte pluridisciplinaire HAL, est destinée au dépôt et à la diffusion de documents scientifiques de niveau recherche, publiés ou non, émanant des établissements d'enseignement et de recherche français ou étrangers, des laboratoires publics ou privés. 
jeudi 17 janvier 2019

Les néonaticides dans la presse française : 357 décès suspects de 1993 à 2012.

Laurence Simmat-Durand, Professeure, Université Paris Descartes, CERMES3, CNRS EHESS UMR 8211, INSERM U 908, Sorbonne Paris Cité

Natacha Vellut, Ingénieure CNRS, CERMES3

Correspondant :

Laurence.simmat-durand@parisdescartes.fr

45 rue des Saints-Pères

75270 Paris cedex 06, France

Tél. 0176533613

Les auteurs déclarent n'avoir aucun conflit d'intérêt. 
jeudi 17 janvier 2019

Résumé

Les néonaticides sont devenus extrêmement rares rendant malaisée la collecte de données judiciaires. Une base de presse a été constituée sur vingt ans afin de tirer parti de la médiatisation de ces affaires, regroupant 357 décès suspects de nouveau-nés sur la période 1993-2012. Cinq résultats ont émergé de cette analyse. Les circonstances de la découverte de l'infraction sont essentielles tout au long de la procédure; Ces affaires sont fortement médiatisées et on assiste sur la période à une « fin » de l'anonymat et de la présomption d'innocence; Une constante historique ensuite qui est la non mise en cause des pères ; L'explication par le déni de grossesse s'amplifie autour de l'affaire Courjeault, pour ensuite s'estomper ; les explications de la mère et sa personnalité ont une influence déterminante sur la peine prononcée.

\section{Abstract}

Nowadays neonaticides are scarce resulting in difficult collection of judicial data. Press articles were recorded on a twenty years period in order to exploit the media coverage around those cases, gathering 357 suspicious neonate deaths from 1993 to 2012. Five results are noticeable. Circumstances of the discovery of the corpses were essential all along the judicial process; these cases received high media coverage and during this period we witnessed the removal of anonymity and end of presumption of innocence; French courts historically chose not to incriminate the fathers; the Courjault case opened the denial of pregnancy outbreak as an explanation before it almost disappeared in the late period; the mothers' explanations and their personalities have a determining influence on the sentences. 
jeudi 17 janvier 2019

Les néonaticides sont devenus extrêmement rares dans les pays industrialisés. Pourtant la presse rapporte ce type d'évènements de façon souvent envahissante, les découvertes de cadavres de nouveau-nés, comme les révélations de supposés meurtres concernant de tout jeunes enfants, constituant l'archétype de l'horrible fait divers, dont la presse régionale, sinon nationale ou internationale, se fait l'écho. Ces articles constituent un matériau peu exploité sur le sujet. Leur utilisation, sur une longue période, permet, au-delà de la faible ampleur du phénomène, de disposer de suffisamment de cas pour valider des hypothèses sur la mise en œuvre de la procédure pénale autour de ces affaires, voire pour faire émerger des hypothèses nouvelles, par exemple sur le rôle éventuel d'une forte médiatisation de certains meurtres.

Les données de la littérature scientifique, basées sur des enquêtes auprès des tribunaux, des services de médecine légale ou des hôpitaux, se fondent sur de faibles effectifs. Les estimations les plus hautes font état de moins d'une vingtaine de cas découverts (par définition, les cas non révélés à la police ou à la justice ne sont pas comptabilisés) par an en France, la quasi-disparition des néonaticides ayant coïncidé avec la diffusion de la contraception puis sa libéralisation, ainsi que celle de l'avortement. Les études populationnelles sont ainsi réduites à quelques dizaines de cas dans chaque pays, même quand de longues périodes de temps sont examinées. Ainsi, l'étude de Putkonen et al. (2007a) en Finlande porte sur 44 cas recueillis sur vingt ans, celle de Shelton et al. (2011) de 1992 à 2005 dans 20 états des Etats-Unis sur 55 cas et celle de Tursz et Cook en France sur 34 cas sur une période de 5 ou 10 ans selon la région (Tursz and Cook 2011). Une étude à partir des données judiciaires à Rio de Janeiro sur près d'un siècle n'avait permis d'identifier que 77 néonaticides (Mendlowicz et al., 1998). Cinq cas ont été extraits de données judiciaires sur les filicides au Québec de 1986 à 1994 (Dubé, Léveillée and Marleau, 2003). Plus récemment, 11 cas ont été identifiés à partir de dossiers judiciaires dans 15 cantons germanophones de Suisse sur la période 1980-2010 (Krüger, 2015). Une revue de registre pour la période 1990-2009 n'a permis d'identifier aucun cas clair pour la Norvège, bien que deux cas douteux soient apparus (Ottesen, 2012). Une étude à partir des registres en Autriche et en Finlande de 1995 à 2005 a permis d'identifier respectivement 23 et 5 cas, ce qui se traduit par des taux très contrastés entre les deux pays (Amon et al., 2012).

Les estimations proposées par A. Tursz et al. à partir de données judiciaires sur trois régions françaises permettent de supposer que les infanticides (enfants de moins d'un an) sont fortement sous-évalués en France et que la proportion de néonaticides (nouveau-nés) pourrait atteindre environ 2,1 pour 100000 naissances (Tursz et al., 2010). La notion d'une sous-estimation et cet ordre de grandeur sont retrouvés dans des données concernant la Caroline du nord (Herman-Giddens et al., 2003), le Royaume-Uni (Craig, 2004) ou la Nouvelle-Zélande (Dean, 2004).

La médiatisation est forte autour des affaires de néonaticides multiples ou de " bébés congelés ", en particulier dans les journaux régionaux, autour de quelques cas de notoriété internationale, comme I'affaire Courjault ${ }^{1}$ ou celle très récemment jugée de Dominique Cottrez ${ }^{2}$. L'analyse approfondie de

\footnotetext{
${ }^{1}$ Véronique Courjault a été condamnée en 2008 à huit ans d'emprisonnement pour l'assassinat de deux de ses enfants retrouvés dans un congélateur à Séoul par son mari, tandis qu'elle était rentrée en France pour ses vacances. Elle a durant le procès avoué avoir également tué un premier enfant en France, mais aucune trace du corps n'avait subsisté.
} 
jeudi 17 janvier 2019

quelques cas d'infanticides à partir de l'examen de la presse a déjà donné des résultats sur le traitement judiciaire des femmes filicides (Rapaport, 2006) mais I'utilisation de la presse de manière exhaustive comme source de données n'a été tentée que dans le cas du Japon, sur l'ensemble des meurtres par leurs parents d'enfants de moins de quinze ans (Yasumi and Lageyama, 2009).

Nous nous proposons d'analyser ici plus de trois cent cinquante cas de décès suspects de nouveaunés rapportés dans la presse française, sur une période de vingt ans, de 1993 à 2012, afin d'envisager le traitement judiciaire qui leur est réservé, les protagonistes des affaires judiciaires et d'explorer les facteurs qui ont pu influencer la peine prononcée comme les " explications » données à ce crime, les circonstances du décès de l'enfant ou l'impact de la médiatisation.

\section{Aspects méthodologiques}

Le recueil a porté sur les articles parus dans la presse française nationale (quotidiens, hebdomadaires, mensuels) ou régionale (61 quotidiens régionaux explorés via des bases d'articles ou leur site internet), relatant le décès suspect ou violent d'un enfant de moins d'un an (plus de 900 enfants décédés sont décrits sur cette période), exception faite des accidents de la circulation routière et des décès par maladie. Parmi ceux-ci, nous avons retenu ici tous les enfants décédés de 1993 à 2012, âgés de quelques heures. Les faits relevés sont les décès de mort suspecte d'enfants à la naissance, ayant entraîné au moins une enquête policière et ensuite l'ouverture d'une information judiciaire, par exemple une enquête pour déterminer les causes de la mort. Les décès des années 2013 à 2015 n'ont pas été inclus bien que recueillis, la durée des procédures ne permettant pas que nous disposions d'un nombre substantiel de décisions les concernant. Les articles ont été identifiés à partir de mots clés spécifiques comme infanticide, découverte de cadavre de nouveau-né ou de nourrisson, "homicide et bébé " ou non spécifiques comme "bébé mort " dans les supports d'actualités. Les archives électroniques des quotidiens ont été interrogées ; les années disponibles sont assez variables et la période 1995-2000 est plus difficile d'accès. La base Factiva a également été utilisée car elle contient les dépêches de l'AFP (Agence France Presse) permettant d'identifier les faits divers tels qu'ils parviennent aux rédactions des quotidiens, et ce depuis 1994 (et plus ponctuellement, la base Europress).

Au total, pour la période 1993-2012, nous disposons de 3914 articles relatifs à des infanticides dont 2292 sur des néonaticides présumés, en langue française. Une base de données a été créée sous Nvivo9, collectant le texte de tous les articles. L'unité de compte choisie (cas) est le nouveau-né, référencé par le nom de la commune et l'année de son décès. Les affaires multiples, comportant une seule mère et plusieurs enfants décédés, comprennent autant de cas que d'enfants décédés, permettant de décrire précisément la mère à chaque décès (son âge a changé, éventuellement son conjoint, sa situation professionnelle, etc.) et les différents enfants (date de décès et de découverte, sexe, mode opératoire). Les décisions judiciaires ne sont par contre comptabilisées qu'une seule fois par mère, celle-ci étant mise en cause ou condamnée pour la totalité de l'affaire et non séparément

\footnotetext{
${ }^{2}$ Dominique Cottrez a été mise en examen en août 2010 après la découverte de huit cadavres de nouveau-nés à son ancien domicile. Elle a été condamnée à neuf ans de prison par la Cour d'assises en juillet 2015.
} 
jeudi 17 janvier 2019

pour chaque nouveau-né tué (comme ce peut être le cas aux Etats-Unis ${ }^{3}$ ). Les cas des autres enfants sont alors indiqués comme faisant l'objet d'une jonction.

Le recueil a été exhaustif pour les cas retrouvés, sauf pour deux affaires très médiatisées au niveau international, celle de Véronique Courjault, qui a tué trois nouveau-nés dont deux à Séoul et celle de Dominique Cottrez, qui a tué huit enfants, pour lesquelles le recueil a été limité à 134 articles pour la première et 318 pour la seconde, en éliminant les articles dupliqués par différents quotidiens, ou repris à l'identique de la dépêche AFP.

L'imprécision de certains articles, qui n'explicitent ni la date du décès ni le lieu exact, " près de Lille ", " en Bretagne » a rendu l'exercice particulièrement délicat. En général, la précision augmente au fur et à mesure de la progression de l'enquête et des recoupements soigneux ont permis d'agréger les articles de chaque cas. Les affaires médiatisées le sont généralement à différents moments, lors de la découverte du cadavre ou du décès, lorsque des éléments de l'enquête paraissent intéressants (analyses génétiques, autopsies), lorsqu'une personne est interpellée puis mise en examen, quand il y a incarcération ou sortie de prison, et enfin au moment du procès. Selon l'importance de l'affaire au niveau local, des journalistes vont ou non assister à la totalité des audiences et rendre compte des débats.

Dans un deuxième temps, ces cas ont été indexés par lecture attentive de tous les articles afin de renseigner 55 variables (caractéristiques) pour chaque enfant/cas, selon les données disponibles ${ }^{4}$. Ces caractéristiques ont été exportées dans un logiciel d'analyse statistique Modalisa6 ou SPSS18 pour en proposer une analyse quantitative. Une analyse de contenu de la presse permet une approche qualitative que nous illustrons par les verbatim des articles cités.

La découverte du décès suspect d'un nourrisson entraîne l'appel à la police ou éventuellement aux pompiers, qui préviendront à leur tour la police. Les articles de presse quand ils mentionnent leur source indiquent ainsi toujours une source policière ou judiciaire, les hôpitaux ne communiquant jamais directement en France sur les suspicions de meurtre. "Huit cadavres de nouveau-nés ont été retrouvés dans un pavillon et un jardin du village de Villers-au-Tertre (Nord) et leurs parents ont été placés mardi en garde à vue, a-t-on appris mercredi de source judiciaire ». (AFP, 28 juillet 2010 18:52). Nouvelles façons de communiquer obligent, dans la dernière affaire de quintuple infanticide à Louchats, découverte en mars 2015, le point sur l'avancée de l'enquête est publié sur la page Facebook de la Gendarmerie, permettant que des éléments précis soient repris par les divers quotidiens.

Les articles de presse relatent les affaires sous une forme très proche de ce que l'on trouve dans les procès-verbaux policiers ou dans les dossiers judiciaires : les informateurs utilisent la structure de la source "officielle" pour donner les éléments à la presse. Selon les lieux ou des circonstances considérées comme plus graves ou plus spectaculaires, il n'est pas rare que ce soit le Procureur de la

\footnotetext{
${ }^{3}$ Une américaine de 40 ans vient d'être condamnée à 30 ans de prison pour avoir tué six nouveau-nés à la naissance alors qu'elle avait déjà trois enfants. Elle a plaidé coupable et les procureurs avaient proposé que les peines de cinq années (par enfant) soient concurrentes. Le juge a refusé indiquant que ce n'était pas possible en matière criminelle. Elle doit donc passer trente ans incompressibles derrière les barreaux. (The Salt Lake Tribune, 20 avril 2015).

${ }^{4}$ Ce qui était également le cas à partir des dossiers judiciaires, un dossier classé sans suite de quelques pages voire paragraphes ne comportant que de rares éléments comparé à un dossier d'instruction de plusieurs années consulté dans une cour d'assises après le procès.
} 
jeudi 17 janvier 2019

République ou son substitut qui fasse une déclaration à la presse, voire soit interviewé par la télévision: "Une femme de 34 ans est soupçonnée d'avoir tué cinq de ses nouveau-nés dont les restes ont été retrouvés mercredi soir dans la cave d'un immeuble de Valognes, au sud de Cherbourg (Manche), une affaire toutefois encore pleine de "zones d'ombres" selon la justice. "L'enquête s'annonce complexe dans cette affaire de cinq infanticides apparents", a estimé jeudi soir le procureur de Cherbourg Michel Garrandaux lors d'un point de presse. Il a précisé que la femme, qui était jeudi soir en garde à vue avec son concubin actuel et son précédent compagnon, avait reconnu être la mère des cinq bébés dont elle a "accouché seule entre août 2000 et février 2006"» (AFP, 18 octobre 2007).

Quand l'affaire est peu suivie par la presse, n'y figure que la simple mention de la découverte d'un cadavre de nouveau-né et des informations importantes peuvent manquer, comme le sexe de l'enfant par exemple. II arrive aussi que dans l'attente de l'ouverture d'une information judiciaire, la presse reste très imprécise sur la commune concernée, surtout quand elle est petite.

Au final, nous disposons de 357 décès suspects d'un nouveau-né de quelques heures, répartis en deux grandes entités: des découvertes de cadavres non élucidées $(n=94)$ et des affaires pour lesquelles une personne, le plus souvent la mère, a été mise en cause $(n=263)$. La figure 1 en indique la répartition par année de 1993 à 2012. S'agissant de petits effectifs, les variations d'une année à l'autre sont importantes et l'année 1998 en particulier intrigue. A titre de comparaison, la figure 2 a été construite à partir des données des services de police et de gendarmerie, portant sur les faits constatés de meurtres de mineurs de quinze ans : on y constate les mêmes types de variations. La courbe en bas correspond aux condamnations portées au casier judiciaire. De plus amples commentaires de ces données disponibles et de leur évolution ont été précédemment publiés (Simmat-Durand, Vellut and Tursz, 2012).

Si dans $87,8 \%$ des cas, un seul enfant est concerné, $12,1 \%$ portent sur un néonaticide multiple, comportant deux à huit enfants décédés au long des années ou simultanément (10 affaires, soit $2,7 \%$ du total des cas sont des jumeaux). Ces affaires révèlent en général la totalité des cadavres, puisque les meurtres successifs des nouveau-nés n'ont pas été découverts au moment des faits, sauf dans trois cas de récidive légale sur lesquels nous reviendrons.

\section{Les étapes de la procédure pénale}

La presse fournit des éléments qui permettent de retrouver la manière de travailler de la justice pour caractériser l'infraction, identifier l'auteur, engager les poursuites et sanctionner les auteurs.

\section{La caractérisation du crime ou du délit}

Comme décrypté à partir des données judiciaires (Simmat-Durand, Vellut and Tursz, 2012), la première étape consiste à mettre en évidence l'existence d'une infraction pénale, que ce soit un meurtre de nouveau-né, un homicide involontaire ou une privation de soins. Si aucune infraction ne peut être retenue, les poursuites ne peuvent être engagées. 
jeudi 17 janvier 2019

Lors de la découverte du cadavre, le Procureur de la République est immédiatement saisi, puisqu'il s'agit jusqu'à preuve du contraire d'une possible affaire criminelle (normalement les femmes accouchent dans un hôpital, ou appellent les secours dans un cas de force majeure) : tout nouveauné voire fœtus jeté dans la nature, à la poubelle, etc., d'autant plus quand la mère n'a pas fait suivre médicalement sa grossesse, constitue une mort suspecte nécessitant une ouverture d'instruction en recherche des causes de la mort.

La question de la viabilité et de la vitalité de l'enfant est essentielle puisqu'elle permet de constituer le crime (Simmat-Durand, Vellut and Tursz, 2012). Si l'enfant était viable et a respiré, le meurtre peut être constitué et les autopsies ou examens complémentaires doivent fournir ces éléments. La mention que l'enfant était viable est absente dans $42,7 \%$ des 357 cas, mais dans seulement $34,4 \%$ des cas quand l'affaire est élucidée et dans $18,6 \%$ des cas quand elle est décrite jusqu'à son terme. Le fait que l'enfant ait respiré est moins souvent mentionné, dans seulement une affaire terminée sur deux. La suite judiciaire de l'affaire est significativement associée à ce premier élément : $67 \%$ des affaires où l'enfant a été déclaré viable sont allées jusqu'au procès.

Le fait que l'enfant ait été mort-né, ou qu'il ait semblé tel à la mère, est un argument qu'elle peut invoquer pour expliquer qu'elle ne lui ait pas porté secours : "Laurence Messmer, une jeune femme menue, le visage fin, les cheveux bruns tirés en un petit chignon, la voix douce, reste persuadée qu'elle n'a pas tué son bébé, mais que cet enfant non désiré, dont elle a nié et caché la grossesse, était mort à la naissance " (Sud-Ouest, 23 mars 2000). Quand les résultats de l'autopsie sont confus ou contradictoires et que la mère persiste à déclarer l'enfant mort-né, les poursuites sont plus souvent abandonnées. "À la barre, les présents ont beau arguer qu'ils n'ont pas tous travaillé sur les mêmes données, la confusion s'installe. Au final, on retient l'idée que l'enfant était vivant mais pouvait... paraître mort. Aux yeux de la loi et de l'intention homicide de la mère, ça change tout. Et c'est bien ce que voulait prouver Me Cohen ... " (La Dépêche, 26 octobre 2010). L'argument que l'enfant était mort à la naissance est mentionné de façon historique dans les études sur l'infanticide, comme élément de défense de la mère (Tillier, 2001). Il peut être aujourd'hui discuté du fait du suivi obligatoire des grossesses en invoquant une responsabilité de la mère dans le non-suivi médical, ayant entraîné une perte de chance pour son enfant.

Néanmoins, des gestes homicides peuvent être retenus à l'encontre de la mère, même si elle affirme que l'enfant n'était pas en vie: "Dans ses aveux, Catherine Guay avait indiqué que l'enfant ne " bougeait pas trop " avant de dire que c'est peut-être elle qui tremblait. "Je lui ai serré le cou mais le bébé n'a pas bougé. "I n'y a pas eu de cris. II n'était pas en vie, ce n'est pas possible ", a-t-elle affirmé aux jurés ${ }^{5}$ " (L'Est Républicain, 11 février 2015).

Enfin, certaines affaires où le cadavre n'a pas été retrouvé vont aller jusqu'au procès, quand bien même cette preuve de vie est manquante: "Une mère de famille, accusée du meurtre de son nouveau-né, a été condamnée à six ans de prison dans la nuit de vendredi à samedi par les assises du Gers, malgré ses dénégations et le fait que le corps n'ait jamais été retrouvé, a-t-on appris auprès de I'une des parties. Audrey Pujos, 35 ans, s'est toujours défendue d'avoir tué la fillette dont elle a

\footnotetext{
${ }^{5}$ « Catherine Guay a été condamnée à cinq ans d'emprisonnement, dont trois avec sursis et mise à l'épreuve, pour le meurtre de son bébé étranglé alors qu'elle venait d'accoucher seule chez elle, à Marseille (notre édition du 10 février). Une peine couverte par sa détention provisoire » (L’Est Républicain, 11 février 2015).
} 
jeudi 17 janvier 2019

accouché le 3 septembre 2011 avec l'aide d'une collègue dans les toilettes du supermarché de Lombez où elle travaillait, et qu'elle dit avoir jetée à la poubelle " (AFP, 15 février 2014).

Toujours concernant l'état de l'enfant à la naissance, un cas particulier d'infraction mal constituée est à relever, lié au terme de la grossesse. Lorsque l'enfant n'est pas né à terme (âge auquel il est présumé viable), sa plus ou moins grande prématurité va attester d'une fausse couche ou tout au moins de circonstances ne permettant pas sa survie hors d'un contexte hospitalier. La prématurité n'est pas toujours rapportée non plus, seuls $6,4 \%$ des enfants sont explicitement désignés comme prématurés ${ }^{6}$. Les données ne permettent pas d'établir un lien entre la prématurité et l'issue de la procédure.

Enfin, pour éventuellement poursuivre, il faut que la découverte du crime se situe en deçà du délai de prescription, soit habituellement dix ans en matière criminelle. Deux remises en question de ce délai, totalement opposées, ont été observées.

Le premier cas consiste à se débarrasser de la qualification de crime, en affirmant dès le départ qu'il s'agit d'un homicide involontaire, matière correctionnelle, pour laquelle le délai de prescription est de trois ans. "...En ouvrant le congélateur, il découvre trois sacs plastiques contenant les corps des nourrissons. Trois jours plus tard, la mère de 40 ans est incarcérée et, faute de preuves, poursuivie pour "homicide involontaire ". La prescription étant dans ce cas de trois ans et les faits datant de 1994, le juge prononce un non-lieu en 2002 » (AFP, 13 octobre 2006).

Au contraire, l'affaire Cottrez, parce qu'elle était exceptionnelle, huit nouveau-nés ayant été tués, a accumulé tous les recours possibles, jusqu'à la cour de Cassation, pour voir modifier la jurisprudence en considérant que le délai de prescription du crime ne court que de la découverte des cadavres et non du meurtre lui-même. "La naissance de sept enfants date d'avant mai 2000, soit plus de dix ans - le délai de prescription en matière criminelle - avant la découverte des premiers corps le 24 juillet 2010. Un "doute" subsiste pour le huitième bébé, né, selon les experts, entre mai-juin et septembreoctobre 2000. L'assemblée plénière de la Cour de cassation, sa formation la plus solennelle, a confirmé le renvoi de Mme Cottrez devant les assises en jugeant que son "obésité", qui avait rendu ces grossesses indécelables par son entourage, avait constitué "un obstacle insurmontable à l'engagement des poursuites". "De ce fait, le délai de prescription s'est trouvé suspendu jusqu'à la découverte des corps", ajoute dans un communiqué la haute juridiction qui, comme l'avait préconisé l'avocat général, a rejeté le pourvoi de Mme Cottrez » (La Dépêche, 7 novembre 2014).

Cette décision a été diversement relayée par la presse, certains médias considérant que la plus haute juridiction s'est ainsi placée sur un plan moral plutôt que sur un plan pénal. Nous y reviendrons.

\section{L'élucidation et la mise en examen d'un(e) suspect(e)}

Une fois l'infraction constituée, son imputation à un auteur ou la mise en cause d'un suspect permet la poursuite de l'instruction. Certaines découvertes de cadavres de nourrissons ne sont jamais élucidées, malgré des recherches longues et fastidieuses (Simmat-Durand, Vellut and Tursz, 2012). Les circonstances de la découverte du cadavre vont jouer un grand rôle dans la suite judiciaire de

\footnotetext{
${ }^{6}$ Soit à peu près la moyenne française qui est de $7 \%$ selon l'Enquête Nationale Périnatale 2010.
} 
jeudi 17 janvier 2019

l'affaire. La non-élucidation de l'affaire est significativement liée à un corps retrouvé dans la nature, les bois ou dans une pièce d'eau, dans un délai variant entre un et sept jours ( $61 \%$ des cas non élucidés). Au contraire, l'élucidation est majoritaire dans les affaires débutant par l'hospitalisation de la mère ( $32 \%$ des affaires élucidées) mais aussi lorsque le corps a été dissimulé dans ou à proximité du domicile et que l'enfant a été étranglé ou asphyxié.

Les articles de journaux cherchent généralement à passionner le lectorat, donc à construire une histoire autour de cette découverte, d'autant plus lorsqu'il s'agit d'une petite ville où les gens se connaissent. "Dans le village ça papote, des ragots, des bruits... Jeudi dernier, à 18h15, le Rhône a laissé flotter I'horreur jusqu'à Lucey, commune paisible de 285 âmes, délimitée par le fleuve et des rangées de vigne. Ce soir-là, un adolescent, 13 ans, a découvert le cadavre d'un nouveau-né dans un sac-poubelle affleurant à la surface du cours d'eau. » (Le Dauphiné, 16 septembre 2009).

Parfois, les enquêtes sont très longues et ne débouchent sur rien, tant il est improbable de découvrir la mère d'un nouveau-né jeté dans la nature, quand bien même des moyens importants sont déployés et que l'autopsie a confirmé le meurtre. Dans le cas ci-dessus, les recherches ont abouti : "La mère d'une nouveau-née, dont le corps a été retrouvé dans un sac en septembre dernier près d'un bras du Rhône en Savoie, a été confondue par son ADN et placée en garde à vue aujourd'hui, a-ton appris auprès du parquet. "Les prélèvements effectués sur le corps de la nouveau-née nous ont permis de mettre en évidence un ADN que nous avons ensuite comparé à celui du voisinage, prélevé lors de l'enquête", a expliqué le procureur de la République de Chambéry, Jean-Pierre Valensi » (AFP I Le Figaro 09/03/2010).

Les 263 cas élucidés de notre corpus correspondent à 228 mères, du fait des néonaticides multiples. Parmi les 230 personnes identifiées comme responsables du décès, 220 mères seules, deux pères seuls, deux couples, deux grands-mères et un couple de grands-parents. Au total, ce sont donc 222 mères qui sont mises en cause.

\section{Les circonstances aggravantes}

Les violences et la préméditation constituent les principales circonstances aggravantes susceptibles d'être retenues dans les affaires examinées, outre la récidive légale que nous examinerons ensuite.

La littérature rapporte que dans le cas des néonaticides, l'enfant est plus souvent tué par inaction que par violence, contrairement aux autres infanticides (Shelton et al., 2011). Dans notre base de données, la façon dont l'enfant a été tué est mieux connue quand l'affaire a été en partie élucidée, ne serait-ce que parce que la mère est entendue et qu'elle a pu fournir des précisions. Néanmoins, le plus souvent une autopsie est ordonnée et la presse en rapporte les principaux résultats dans les jours qui suivent. Dans la moitié des cas non élucidés, on ne dispose pas du mode opératoire. "L'autopsie pratiquée hier à la demande du parquet de Guingamp devait révéler sur le corps des traces de coups portés à la tête et de strangulation, confirmant une " manifestation externe humaine " qui aurait provoqué le décès du bébé. Après avoir ouvert une information judiciaire pour meurtre sur enfant de moins de 15 ans, le parquet de Guingamp s'est dessaisi hier en fin de journée au profit du pôle de l'instruction de Saint-Brieuc » (Le Parisien, 26 mars 2008). 
jeudi 17 janvier 2019

Le plus souvent, l'enfant est étranglé ou asphyxié ( $34 \%$ du total et $43 \%$ des cas où on connaît le mode opératoire), notamment en le plaçant dans un sac plastique, en l'étranglant avec les mains ou un objet. "Valognes : la mère avoue avoir tué six bébés à la naissance. Céline a expliqué aux enquêteurs avoir à chaque fois accouché seule, puis avoir étouffé les nouveau-nés en "plaquant sa main sur le visage de l'enfant afin de l'empêcher de respirer", selon le procureur. Deux d'entre eux ont également été "étranglés avec une cordelette". (AFP, 19 octobre 2007). Ensuite, $14 \%$ des enfants sont noyés, le plus souvent dans les WC ou la baignoire, soit parce que la mère a accouché sur les toilettes et y a laissé l'enfant, soit parce qu'elle l'a activement noyé. "Jeudi, les débats avaient permis aux jurés de cerner le terrible cheminement qui a conduit cette Berruyère de 29 ans à commettre l'irréparable, le 20 juillet 2004 : accoucher seule chez elle dans sa salle de bains et retourner le bébé dans la cabine de douche afin qu'il meure " (La Nouvelle République du CentreOuest, 25 septembre 2006). Enfin, $14 \%$ des nouveau-nés sont activement agressés, soit par des coups, ou par arme ou enfin défenestrés. "Sandra Kauffmann, fille d'un père français et d'une mère d'origine algérienne, célibataire au moment des faits, avait accouché dans les toilettes du domicile familial à Dietwiller, près de Mulhouse, mis le nouveau-né dans un sac poubelle et lui avait porté des coups avec un instrument piquant et tranchant parce qu'il bougeait encore » (AFP, 1 octobre 1997).

Il faut a contrario distinguer le défaut de soins où l'enfant est abandonné à son sort, sans que le cordon ombilical ne soit coupé : il est laissé sur le lieu de l'accouchement ou dans le même secteur. "Elle a dit aussi qu'elle était, à chaque fois après ses accouchements clandestins de 2003 et 2005, revenue voir 48 heures après dans quel état se trouvaient ses bébés. Et les deux fois, elle aurait constaté leur mort ». (La Montagne, 4 février 2009). Une variante est le délaissement de l'enfant, c'est-à-dire le fait de le déposer dans un lieu où il ne peut lui être porté secours dans un délai permettant sa survie : dans un champ, dans une poubelle, dans un lieu public de nuit, etc.

Cette distinction entre meurtre actif et par inaction a une influence indéniable sur l'issue de la procédure puisque l'affaire se solde deux fois plus souvent par un procès ( $49 \%$ versus $24 \%, p<0,001)$ quand l'auteur a activement cherché à tuer l'enfant ou a fait preuve de violences à son égard.

Du point de vue de l'action judiciaire, une autre circonstance aggravante sera la préméditation, ce qui distinguera les coups et blessures ayant entraîné la mort, ou les délaissements ayant entraîné la mort, des homicides et des meurtres aggravés. Historiquement, l'infanticide (néonaticide) était assimilé à un assassinat, puisque la mère avait eu toute sa grossesse pour le préparer, d'où les débats en 1810 pour lui appliquer la peine de mort (Geoffroy-Poisson, 2005). Aujourd'hui, la littérature admet généralement que l'acte est rarement prémédité, la mère agissant sous le coup de la peur, du choc et de la culpabilité (Shelton et al., 2011), d'où des peines modérées dans grand nombre de pays européens (Putkonen et al., 2007a).

De nombreux écrits montrent la confusion entre les signes de l'accouchement et des douleurs digestives, amenant la mère à accoucher dans les toilettes. Pourtant, un certain nombre de femmes vont préparer l'accouchement et la mort de l'enfant et les néonaticides multiples ne rentrent pas dans cette explication d'une mère prise par surprise ou sous le coup de la panique. "Après un séjour en hôpital psychiatrique, cette mère, interpellée lundi, a avoué, lors de sa garde à vue, qu'après avoir dissimulé sa grossesse à son entourage, elle avait envisagé de tuer son bébé. Elle avait d'ailleurs emporté un couteau et une pelle pour pouvoir l'enterrer" (AFP, 14 janvier 2004). Les décisions judiciaires que nous allons examiner reflètent ce point. 
jeudi 17 janvier 2019

Pour les affaires décrites au XIXème siècle, la dernière circonstance aggravante retenue était la vie désordonnée de la mère (Geoffroy-Poisson, 2005) et plus particulièrement la question des naissances illégitimes (hors mariage). Cette question n'est plus d'actualité, bien que quelques éléments sur le mode de vie de la mère puissent être évoqués ( $c f$. infra).

\section{Absence de poursuites ou silence de la presse?}

Les suites de l'affaire sont connues essentiellement quand l'affaire a été élucidée. Quand un cadavre a été découvert et qu'aucun suspect n'a pu être identifié, neuf fois sur dix, il n'y a pas d'article de presse supplémentaire et la procédure restant contre $X$, on peut en déduire qu'elle a été classée sans suite. Dans la recherche précédente sur les dossiers judiciaires, les néonaticides non élucidés ont été pour moitié classés sans suite et pour moitié l'objet d'une ordonnance de non-lieu. Si effectivement les affaires concernées sont restées non élucidées, l'abandon des poursuites est l'hypothèse vraisemblable.

Quand la mère ou un autre auteur a été identifié, la moitié des affaires $(49,4 \%)$ est décrite jusqu'au procès, auxquelles s'ajoutent les jonctions $(8,7 \%)$ concernant les décès multiples. Des non-lieux ont été prononcés dans $6 \%$ des cas. Restent donc $35 \%$ d'affaires pour lesquelles l'identification a eu lieu et dont nous n'avons pas la suite. Parmi ces affaires, un quart concerne des faits des années 2010 à 2012 pour lesquelles potentiellement le procès n'a pas encore eu lieu (par exemple en février 2016, quatre affaires ont été médiatisées lors de leur passage aux Assises parmi celles de notre corpus pour des faits datant de 2010 à 2012). Ce sont donc moins de $30 \%$ des affaires élucidées antérieures à 2010 qui n'ont pas été jugées. Nous avons ainsi proportionnellement plus de poursuites que ce qui avait été observé dans l'enquête auprès des tribunaux.

Penser que la presse rapporte tous les procès et néglige les classements sans suite paraît donc envisageable. La vision stéréotypée ou dramatisée portée par la presse de ces mères néonaticides et des sanctions qui leur sont applicables apparaît peu compatible avec l'abandon des poursuites pénales, que la presse ne commente pas.

Une autre piste retient ici notre attention. Quand la mère est mineure (22 cas décrits), la loi interdit que le résultat d'un procès la concernant soit diffusé. Effectivement, la proportion d'affaires sans résultat est double lorsque la mère était mineure au moment des faits, d'autant plus que les circonstances sont souvent assez différentes.

Quelques résultats de classement sans suite sont néanmoins publiés, en particulier quand une autre affaire nécessite de s'intéresser au résultat précédent: "Durant l'enquête, elle avait affirmé avoir enroulé le corps de sa victime dans une serviette, objet que les enquêteurs n'avaient toutefois pas retrouvé. Ils avaient en revanche découvert un tel linge autour du cadavre d'un bébé, retrouvé dans une poubelle six ans auparavant. Le dossier de cet infanticide, jamais élucidé, avait été classé " (AFP 13 novembre 1998). 
jeudi 17 janvier 2019

\section{Les décisions et les peines}

Parmi les affaires où les poursuites ont été engagées, onze se sont terminées par des ordonnances de non-lieux, dont une non élucidée après ouverture d'une instruction, les autres n'ayant pas abouti à un renvoi devant la juridiction de jugement, dont un suicide de la mère en détention provisoire. Dix mères et deux pères sont concernés, une affaire n'ayant pu déterminer qui du père ou de la mère avait finalement tué l'enfant.

L'ordonnance de non-lieu est la décision la plus rarement prononcée, mais elle apparaît surreprésentée quand l'enfant a été reconnu comme mort-né par l'autopsie, après une enquête en recherche des causes de la mort, d'autant plus si d'autres éléments de l'affaire comme la minorité et la panique ont pu intervenir : "Une mère de seize ans, dont le bébé avait été retrouvé mort près d'un immeuble à Belfort, le 20 août 1999, a bénéficié d'un non-lieu. La jeune femme, qui habitait l'immeuble, avait affirmé que son bébé, dont elle avait accouché seule, était mort-né et que, paniquée, elle l'avait jeté par la fenêtre. L'autopsie a confirmé ses dires " (Le Monde, 13 février 2000).

A l'issue de la procédure, 118 cas ont été renvoyés devant une cour d'assises dont un en cour d'assises des mineurs et sept devant un tribunal correctionnel dont un tribunal pour enfants, ce qui signifie qu'ils ont été considérés comme des délits et non comme des crimes : trois pour homicide involontaire, trois pour délaissement de mineur, un pour complicité de meurtre. "Dans la soirée du 3 mars 2002, Céline accouche seule, dans sa chambre. La maman perd beaucoup de sang. "II n'a pas crié, il avait du mal à respirer ", se souvient Céline. Dans un état second, la jeune fille enveloppe le nouveau-né dans une serviette sans toutefois lui recouvrir le visage. Mais ce geste est fatal. "Est-ce que vous saviez ce qu'il fallait faire juste après l'avoir mis au monde? ", interroge la présidente, Brigitte Arnaud-Petit. Le visage couvert de larmes, la jeune fille répond que non. L'expertise médicale stipule qu'avec des soins simples et immédiats, le nourrisson aurait pourtant survécu. .../... Céline est d'abord mise en examen pour homicide volontaire et placée sous contrôle judiciaire. Les premières expertises aboutissent à une requalification du dossier en homicide involontaire pour défaut de soins ". (La Nouvelle République, 14 octobre 2004). Dans le cas ci-dessus, le jeune âge de la mère et son impréparation à la maternité au moment des faits peuvent expliquer la décision, dans une affaire en tous points semblables à d'autres qui ont pourtant été renvoyées en cour d'assises.

La presse rappelle de manière incantatoire à chaque nouvelle mise en examen que la mère encourt la réclusion criminelle à perpétuité. "La prévenue encourt la réclusion criminelle à perpétuité pour infanticide et assassinat sur mineur " (Le Matin, 25 juin 2015). Une telle peine n'a été que très rarement prononcée contre une mère néonaticide depuis le début du $20^{\mathrm{ème}}$ siècle $^{7}$ et cette rigueur de la loi a toujours contrasté avec les peines modérées finalement prononcées (Tinkova, 2005). Des cas de néonaticides multiples ayant entrainé une condamnation à perpétuité sont décrits dans la presse des autres pays européens ${ }^{8}$. Cette sanction maximale est rappelée quelquefois au cours du

\footnotetext{
${ }^{7}$ Dans une étude sur trois régions françaises de 1930 à 1955, aucune mère n'a été condamnée à une peine supérieure à trois années d'emprisonnement ferme. LÉAUTÉ J., 1968, Recherches sur l'infanticide (1955-1965), Paris: Dalloz.

${ }^{8}$ Exemple : «Une Finlandaise condamnée à la perpétuité pour cinq infanticides. internet@lesoir.be (Avec les rédactions du Soir en ligne, du Soir, d'AFP, d'AP et de Belga), 15 juin 2015.

La justice finlandaise a condamné lundi une femme de 36 ans à la prison à perpétuité pour les meurtres entre 2005 et 2013 de ses cinq nouveaux-nés, qu'elle avait cachés dans un congélateur. Le tribunal de Oulu (ouest) l'a
} 
jeudi 17 janvier 2019

procès : "Même si le code pénal prévoit comme peine maximale la réclusion criminelle à perpétuité en cas d'infanticide, "une telle peine n'a pas de sens dans un tel contexte", a estimé l'avocat général Patrick Beau, qui a requis cinq ans de prison assortis d'un sursis " (AFP, $1^{\text {er }}$ octobre 1997). Notre corpus comporte ainsi 321 occurrences du mot perpétuité, montrant l'importance qu'il revêt dans la rédaction des articles.

Cent-quatre mères ont été condamnées à une peine d'emprisonnement. Cette peine d'emprisonnement est ferme sans sursis dans $40 \%$ des cas, mixte dans presque $40 \%$ des cas et avec sursis total dans $20 \%$ des cas. Ces peines vont de six mois avec sursis total, prononcés par un tribunal correctionnel pour homicide involontaire, à 23 ans de réclusion criminelle, dans un cas d'infanticides multiples en état de récidive ( $c f . i n f r a)$, ce qui démontre un large éventail de peines.

La peine d'emprisonnement ferme est en moyenne de 5,35 ans $( \pm 4,15)$. Quand le meurtre a été retenu la peine minimale possible et prononcée est de deux ans. Sur l'ensemble examiné, 15 femmes ont été condamnées à des peines d'au moins dix ans d'emprisonnement, cependant la moitié des faits des années 2011 et 2012 ne sont pas encore arrivés jusqu'au procès. En effet, disposant de la date de la découverte ou du décès du nouveau-né et de la date de la dernière décision judiciaire, on peut calculer qu'une affaire nécessite en moyenne $2,97 \pm 1,4$ ans pour aller à son terme. La plus longue a nécessité 8,4 ans avant d'être jugée définitivement par une seconde cour d'assises, le Procureur de la République ayant fait appel d'un premier acquittement.

Enfin, dix acquittements dont huit concernant des mères ont été prononcés par les cours d'Assises, cinq pour meurtre, un pour violences volontaires et quatre pour délaissement ou privation de soins. De plus, une mère acquittée en première instance a été condamnée par la juridiction d'appel.

Avant de revenir sur ce qui peut influencer les décisions judiciaires, nous allons évoquer les personnes impliquées dans ces affaires.

\section{Les protagonistes}

A la différence d'autres pratiques judiciaires, la mise en cause pour complicité est rare, les mères sont donc seules face à la justice, ce qui a amené à parler de dimorphisme sexuel des accusations pour les périodes du 18è et 19è siècles en France (Tinkova, 2005). Les homicides ont été perpétrés le plus souvent par la mère seule, deux par la grand-mère, un par les deux grands-parents, deux par le père ou beau-père, deux par les deux parents. Dans $5,9 \%$ des cas, la mère a bénéficié d'une complicité pour tuer l'enfant ou dissimuler le corps, le plus souvent de son mari ou de sa mère ou belle-mère.

reconnue coupable de cinq meurtres et d'atteinte au respect dîu aux morts, car elle n'avait pas offert aux nourrissons de sépultures convenables. «Les homicides ont été commis en laissant les nouveaux-nés sans soin ni chaleur (...) manifestement, cette mesure (...) a entraîné une agonie particulière pour les bébés», a indiqué la cour dans son jugement. La femme, qui a un fils de 14 ans, a plaidé non-coupable, affirmant que les enfants étaient mort-nés. Se fondant sur des examens dentaires, le tribunal a jugé que certains des enfants avaient vécu jusqu'à quatre jours avant de mourir dans la bassine dans laquelle ils avaient été déposés » ». 
jeudi 17 janvier 2019

Au sein de notre corpus, 263 cas ont été élucidés ce qui correspond à 228 mères différentes, du fait de 17 infanticides multiples élucidés : 8 mères ont tué deux enfants, 5 trois enfants, les quatre dernières ayant tué respectivement quatre, cinq, six et huit enfants.

\section{Qui sont les mères concernées?}

L'âge de la mère est généralement mentionné et ne manque que dans moins de $6 \%$ des affaires élucidées. L'âge moyen des mères est de $27,58 \pm 8$ ans. On est ici très proche de l'âge moyen à la maternité en France, critère sur lequel ces femmes ne se distinguent pas. Ces données confirment celles obtenues sur des affaires judiciaires en France (Simmat-Durand, Vellut and Tursz, 2012), comme en Suisse (Krüger, 2015) et s'éloignent du profil rapporté pour les pays comme les Etats-Unis ou le Royaume-Uni où les femmes sont très jeunes, par exemple 21 ans dans l'étude de J.L. Shelton et al. (2011), à rapprocher de la part des grossesses chez les adolescentes dans ces pays. Ce constat a également été fait pour des données finlandaises (Putkonen et al., 2007b).

D'ailleurs des différences très significatives $(p<0,001)$ apparaissent entre les femmes ayant moins de 25 ans et leurs aînées. En termes de profils, les femmes de moins de 25 ans sont significativement associées au décès d'un premier enfant, découvert immédiatement après les faits, le plus souvent à la poubelle ou dans une décharge. Les femmes de plus de 25 ans ont quant à elles plus souvent au moins deux enfants précédents et le corps du nouveau-né est découvert après un certain délai (d'autant plus que tous les enfants placés au congélateur sont issus de ces mères).

Compte tenu de cette répartition par âges, $48,7 \%$ des femmes sont mentionnées comme ayant au moins un enfant précédent vivant, $22,6 \%$ en ayant trois ou plus (jusqu'à six enfants vivants). De plus, $10,5 \%$ des femmes sont mentionnées comme ayant eu au moins un nouvel enfant au moment de leur procès, dont $2 \%$ au sein d'un nouveau couple. Le niveau d'études de la mère n'est indiqué que dans $22 \%$ des cas et il n'est pas possible de discerner un niveau d'études spécifique, presque autant de femmes sont déclarées illettrées ou de niveau primaire que de niveau supérieur.

La profession de la mère est l'une des variables la moins bien renseignée, avec $52 \%$ de valeurs manquantes. Pour celles qui sont mentionnées : $37 \%$ sont employées, $26 \%$ étudiantes ou lycéennes, $8 \%$ mères au foyer et $12 \%$ sans emploi. Quand on consulte des dossiers judiciaires, cette variable est également mal renseignée (Tursz et al., 2011). L'impression qui se dégage est néanmoins que les néonaticides sont susceptibles d'advenir dans tous les milieux sociaux, bien que certains écrits penchent sur un lien avec un milieu social pauvre ou déshérité. Si corrélation il y a avec l'extrême misère (Bydlowski, 2011), c'est plutôt d'une misère affective qu'il s'agit.

La recherche d'explications par les journalistes se focalise sur des arguments hors normes, comme des cas sociaux, des enfances difficiles, une violence extrême ou des explications " psy " permettant la mise à distance comme des pathologies psychiatriques ou un déni de grossesse. Les circonstances très atypiques comme les meurtres multiples ( 20 femmes en 20 ans), les nouveau-nés congelés (23 femmes) ou la récidive (trois femmes), sont plus fortement médiatisées, car susceptibles de créer une fascination par l'horreur. 
jeudi 17 janvier 2019

\section{Le père de l'enfant n'est pas inquiété}

Comme retenu dans la littérature, la femme est isolée dans le vécu de ce crime et la mise en cause du père n'a jamais été le choix de la justice française, contrairement à d'autres pays (Tinkova, 2005). De ce fait, on sait très peu de choses des caractéristiques du père, son âge même n'est mentionné que dans $27 \%$ des cas (en moyenne 34,9 \pm 12 ans). Quand on peut calculer l'écart d'âge entre les conjoints, $28 \%$ des femmes ont conçu l'enfant avec un conjoint ayant plus de dix ans de plus qu'elles $^{9}$.

Les circonstances courantes d'une grossesse cachée et non suivie médicalement ont été amalgamées à la notion de déni de grossesse ${ }^{10}$, où personne, pas même la femme, n'est au courant. Dans ce contexte, il n'est pas surprenant de constater que les pères ne sont jamais ou presque "inquiétés ", ni même inquiets, à la suite du meurtre du nouveau-né. Dans de rares circonstances où le père aide la mère ou assiste à la naissance, il peut être mis en cause pour complicité ou non-assistance. "Une information judiciaire va également être ouverte contre le père de la petite fille, née le 16 octobre et décédée le même jour, pour non assistance à personne en danger, a-t-il précisé lors d'une conférence de presse. "Des indices graves et concordants permettent d'affirmer que la mère a volontairement donné la mort à son enfant et que le père n'a pas assisté le nourrisson en péril ", a précisé le représentant du parquet » (Nouvel-Observateur, 19 octobre 2012).

Le père n'a été retenu comme complice des faits que dans $3 \%$ des cas et comme auteur principal que dans deux affaires élucidées, particulièrement sordides, l'une de viols répétés dans le cadre d'un inceste et l'autre de nouveau-né enterré vivant sous les yeux de sa mère. Parmi les sept considérés comme complices, un a bénéficié d'un non-lieu, quatre ont été condamnés à des peines de deux et trois ans d'emprisonnement ferme et deux à une peine avec sursis total. "Une mère et un père de famille ont été respectivement condamnés jeudi à six et trois ans de prison ferme par la cour d'assises du Doubs à Besançon pour avoir volontairement laissé mourir de froid leur nouveau-né enfermé à l'extérieur de leur appartement par une nuit de janvier 2001. Sept ans de prison avaient été requis contre la mère de l'enfant, mise en examen pour meurtre, et trois ans contre le père accusé de privation de soins mortelle » (AFP, 11 septembre 2003).

La littérature internationale rapporte que les cas où le père commet le néonaticide sont rarissimes. De manière générale, la complicité n'est pas retenue dès le début de l'enquête, même si les circonstances globales de l'affaire la rendent hautement probable, comme par exemple cette femme qui affirme avoir fait un déni de grossesse et que son mari n'était pas au courant, alors que le début de la procédure repose sur une dénonciation d'un voisin l'ayant vue enceinte, mais n'ayant jamais vu l'enfant.

Dans de très rares affaires également, plutôt récentes, le père se constitue partie civile et réclame des dommages-intérêts à la mère pour l'avoir privé de sa descendance, voire pour se protéger de toute complicité. « Luc Margueritte, son second concubin, partie civile au procès, s'est dit pour sa part

\footnotetext{
${ }^{9}$ Alors que l'écart d'âge moyen entre conjoints dans les données de l'Insee en population générale n'est que de deux ans.

${ }^{10}$ Cette explication existait déjà dans les études historiques, sans porter ce nom : « les prévenues adoptaient des systèmes de défense stéréotypés ... elles disaient aussi avoir ignoré leur état ou s'être trouvées « surprises » par les douleurs de l'accouchement » TINKOVA D., 2005, "Protéger ou punir ? Les voies de la décriminalisation de l'infanticide en France et dans le domaine des Habsbourg (XVIIIè-XIXè siècles)," Crime, Histoire \& Sociétés $9(2): 43-72$.
} 
jeudi 17 janvier 2019

"partagé" jeudi matin. "Céline m'a fait du mal. Pour moi, elle est coupable. Mais son entourage et moi-même sommes un peu coupables de ne pas avoir vu sa détresse" " (La Croix, 18 mars 2000).

\section{L'existence de l'enfant par le procès pénal}

L'enfant n'est souvent pas reconnu comme tel par la femme néonaticide, jusqu'à ce que la procédure criminelle tout à la fois reconnaisse la femme comme mère et donne une existence à cet enfant (Henry, 2009). Le corps de l'enfant, de déchet, devient un être qui a vécu puis est mort (SimmatDurand and Vellut, 2013) : "Aucun n'a crié. Je leur cachais la tête avec un linge. C'est horrible à dire, mais pour moi ils n'étaient rien. Je ne les avais pas imaginés. C'était comme un engrenage... » (Ouest France, 16 mars 2010).

L'enfant a cependant peu de poids dans le procès pénal, faute d'avoir eu une existence et d'avoir été nommé. Néanmoins, certaines familles se portent partie civile pour le défendre, voire des associations au nom de l'enfant. Sinon, quand il n'est pas oublié dans le procès, c'est le ministère public qui se charge de le représenter ou tout au moins de l'évoquer : " "Je veux rendre justice à ceux qui n'ont pas demandé à naître, ni à mourir dans des circonstances atroces, quatre bébés sans noms, sans acte de naissance", avait déclaré l'avocat général, Jean-Luc Desport, dans son réquisitoire. II a regretté de n'avoir pas entendu Christine F., 41 ans, exprimer "un seul remord en trois jours" de procès » (AFP, 17 novembre 2005).

\section{Quels éléments ont une influence sur la peine prononcée?}

Le corpus réuni permet de disposer d'un nombre important de résultats de mises en examen, soit 11 non-lieux et 130 personnes jugées dont 10 acquittées, permettant de confirmer certaines hypothèses posées à partir de l'étude de 32 dossiers judiciaires et d'en voir émerger de nouvelles.

\section{Les éléments liés à la procédure}

La matière criminelle ou correctionnelle est définie en fonction de la qualification qui sera retenue pour les faits au moment du renvoi devant la juridiction de jugement. Une première qualification est donnée au parquet en début de procédure, dont nous ne disposons généralement pas dans la presse.

La qualification des faits au moment du renvoi devant la juridiction de jugement est largement indiquée, l'information n'étant manquante que dans $7 \%$ des cas. La qualification la plus fréquente à l'encontre de la mère est le meurtre ou homicide volontaire $(58,5 \%)$, éventuellement avec recel de cadavre $(13,6 \%)$, le délaissement d'un mineur suivi de la mort (9\%), la privation de soins ayant entraîné la mort (5\%), I'homicide involontaire (4 \%), l'assassinat ou meurtre avec préméditation (3 $\%$ ) et enfin les coups et blessures volontaires ayant entraîné la mort sans intention de la donner (2 $\%)$. Pour les pères, un assassinat, un homicide volontaire, les autres étant jugés pour privation de soins ou non-assistance à personne en danger, donc comme complices et un pour recel de cadavre. 
jeudi 17 janvier 2019

Nous observons ainsi proportionnellement plus de qualifications de meurtre ou d'homicide volontaire que dans l'enquête à partir des juridictions (Simmat-Durand, Vellut and Tursz, 2012), ce qui pourrait traduire un biais, si la presse se fait plus facilement l'écho des affaires "graves " ou susceptibles de captiver le lectorat. Cela pourrait également traduire des variations régionales ou temporelles puisque nous couvrons toute la France et sur une durée quatre fois plus longue.

La peine ferme prononcée varie en fonction de cette qualification : de 7,4 ans en moyenne pour assassinat et 6,7 pour meurtre à 4 ans pour un délaissement ayant entraîné la mort et 2,5 ans pour des violences ayant entraîné la mort sans intention de la donner. On retrouve la préméditation puis l'intentionnalité comme éléments déterminants de la sanction, éléments qui transparaissaient déjà dans les modes opératoires de la mort du nourrisson et la distinction mort active ou par inaction.

Dans le même ordre d'idée, autant le déni de grossesse, la panique ou d'autres explications de même type apparaissent légitimes quand un seul enfant a été tué, autant quand la mère a tué plusieurs enfants, ces circonstances semblent moins cruciales et sa seule personnalité retient l'attention. Si I'on met en rapport le nombre de nouveau-nés tués et la partie ferme de la peine, la corrélation est positive et significative, même si, comme nous l'avons précisé, la sanction française n'est pas multipliée par le nombre d'enfants tués.

La loi française prévoit par contre une aggravation des peines en cas de récidive. Le risque de récidive au sens légal du terme, c'est-à-dire une nouvelle infraction pénale après une première condamnation, est considéré comme très faible dans les affaires de néonaticide, les poursuites judiciaires, la sanction et souvent le travail thérapeutique entamé amenant la mère à réaliser la gravité de son acte. Ainsi Philip Resnick dans une revue de littérature internationale, note : " $L a$ majorité des femmes commettent un néonaticide dans le contexte de situations désespérées et non en raison d'un personnalité mauvaise. Elles ne sont donc pas susceptibles de commettre d'autres crimes » (Resnick, 2009).

Le mot récidive est souvent improprement employé dans la presse pour désigner les néonaticides multiples, donc une réitération, où tous les cadavres sont découverts à une même date. $\mathrm{A}$ propos d'une affaire, un expert psychiatre devant la cour d'assises reconnaît que ce risque existe : "Le risque de récidive existe, les conditions peuvent se représenter pour passer à l'acte, si elle se retrouve isolée " (La Nouvelle République, 3 février 2011). Certaines femmes, face à ce risque, prennent des décisions extrêmes en se faisant ligaturer les trompes : "Sophie Villaron, qui s'est fait stériliser à sa demande pendant sa détention préventive, "a besoin d'un suivi socio-judiciaire suffisamment long pour se soigner même si le risque de réitération est à exclure", a affirmé dans son réquisitoire Mme Assonion (avocate générale) » (La Provence 13 octobre 2011).

Seules trois affaires relèvent d'une récidive légale dans notre corpus, la mère ayant été condamnée une première fois et ayant de nouveau tué un ou des enfants. Le cas le plus récent est celui-ci : "Bébés congelés à Ambérieu : la mère déjà condamnée pour des faits similaires. En 2002, la jeune femme avait accouché d'un bébé qu'elle avait demandé à sa mère d'abandonner dans un sac. La mère de deux foetus ou nouveaux-nés congelés retrouvés dimanche à son domicile à Ambérieu, dans l'Ain, avait déjà été condamnée en 2005 à quinze ans de prison après avoir tué son nouveau-né, avec l'aide de sa mère, rappelle le journal régional Le Progrès dans son édition de mardi» (AFP, 26 mars 2013 à 10:21). La nouvelle affaire est découverte en 2013, pour des faits de 2011 et 2012 (deux nouveau-nés tués); cette circonstance a nécessité une explication de la procédure: "Selon le 
jeudi 17 janvier 2019

procureur Denis Mondon, ... elle était restée derrière les barreaux de juillet 2002 à décembre 2010. Le procureur a expliqué que, justifiant d'un emploi et d'une obligation de soins, elle avait bénéficié d'une libération anticipée. "Madame C. a purgé toute sa peine. Elle est restée détenue huit ans, quatre mois et neuf jours. Et la cour d'assises disait dans son jugement qu'elle pouvait avoir un aménagement de peine après sept ans et six mois», a-t-il ajouté » (Le Parisien, 26 mars 2013). La deuxième comparution devant les assises, en état de récidive, vaudra à Audrey Chabot une réquisition de 27 années de réclusion criminelle et une condamnation à 23 années en mars 2015.

Le quantum ferme prononcé par la juridiction peut être influencé par la durée passée en détention provisoire. Deux tiers des mères ont été placées en détention provisoire lors de leur mise en examen. La durée moyenne observée a été de 12 mois, le maximum étant de 54 mois. Pour éviter les recours ultérieurs, la mise en détention provisoire implique généralement une peine d'emprisonnement ferme. "La cour d'assises de Seine-et-Marne a condamné mardi une mère de famille accusée d'avoir tué son nouveau-né en avril 1999 à Saint-Fargeau-Ponthierry à cinq ans de prison dont 9 mois ferme déjà couverts par la détention provisoire " (AFP, 27 mai 2003). Néanmoins, deux non-lieux et cinq acquittements ont été prononcés pour des femmes ayant effectué de la détention provisoire. Dans un autre cas, la jeune femme de 20 ans a été acquittée, alors que l'avocat général avait requis 17 mois d'emprisonnement ferme pour couvrir la durée de sa détention effectuée en préventive. Dans cette affaire, le doute était permis sur le fait que ce soit elle qui ait égorgé le nouveau-né et non son père à elle, non mis en cause dans la procédure.

Enfin, le prononcé de la peine doit prendre en compte une éventuelle abolition ou altération du discernement ${ }^{11}$. Si l'abolition du discernement, qui peut inciter la justice à conclure à une irresponsabilité pénale est très rare dans les affaires de néonaticide, la reconnaissance de l'altération du discernement est par contre fréquente, selon une étude réalisée à partir d'expertises judiciaires. Cette reconnaissance protège, comme le législateur le prévoyait, des peines les plus lourdes (Vellut and Simmat-Durand, 2013). Cette notion peut être systématiquement employée dans d'autres pays où le législateur considère que les circonstances d'un accouchement imprévu et solitaire sont de nature à altérer le discernement (Putkonen et al., 2007a).

On sait peu de choses précises sur l'état de santé mentale de la mère, bien que quelques articles mentionnent que cette question a été soulevée lors du procès. Pour 19 mères, il est indiqué qu'une altération du discernement a été mentionnée, pour huit mères qu'elles étaient dépressives, pour six qu'elles souffraient d'une pathologie psychiatrique, pour une qu'elle était simple d'esprit, et pour une autre qu'elle était sous curatelle. Deux femmes ont été internées d'office en cours de procédure et deux se sont suicidées, une au moment des faits et l'autre en détention provisoire.

II n'est fait mention d'une abolition du discernement que dans trois cas, dont un terminé par une peine de cinq ans avec sursis total et deux par un acquittement, dont un sera remis en cause après appel du ministère public. "JUSTICE L'abolition totale du discernement de la jeune femme, qui a accouché seule dans une résidence agenaise en 2012, a été établie ; Non-lieu dans l'affaire de la mère

\footnotetext{
${ }^{11}$ L'article 122-1 de la loi no $92-683$ du 22 juillet 1992 portant réforme des dispositions générales du code pénal, prévoit : « Alinéa 1 : N'est pas pénalement responsable la personne qui était atteinte, au moment des faits, d'un trouble psychique ou neuropsychique ayant aboli son discernement ou le contrôle de ses actes. Alinéa $2:$ La personne qui était atteinte, au moment des faits, d'un trouble psychique ou neuropsychique ayant altéré son discernement ou entravé le contrôle de ses actes demeure punissable ; toutefois, la juridiction tient compte de cette circonstance lorsqu'elle détermine la peine et en fixe le régime ».
} 
jeudi 17 janvier 2019

infanticide " (Sud-Ouest, 8 août 2014). L'altération du discernement concerne 17 cas de meurtre, un assassinat et un cas de violences volontaires ayant entraîné la mort sans intention de la donner. Une femme a bénéficié d'un non-lieu. Trois femmes ont été condamnées à des peines d'emprisonnement ferme de huit, neuf et quinze ans, six femmes ont été condamnées à des peines avec sursis total et neuf à des peines de cinq ans d'emprisonnement avec de 2 ans à 4 ans et 9 mois de sursis.

La presse mentionne essentiellement les dossiers où la question de l'abolition du discernement a été centrale ou ceux pour lesquels les experts psychiatriques se divisent, laissant les jurés apprécier. "Meurtre. Après des divergences d'experts, la cour d'assises a choisi son camp. Elle n'a pas dit que Cécile avait étranglé Quentin. Mais que cette femme de trente-sept ans l'avait asphyxié en le plaçant volontairement dans un sac plastique.../... Discernement. Les jurés ont surtout reconnu que son discernement avait été aboli au moment de l'accouchement. Ce qui la rend irresponsable pénalement. Ce point a divisé les trois expertises psychiatriques. La première pensait le discernement aboli. La deuxième établissait "une large altération" du discernement. Et la troisième "une altération modérée à moyenne » (Le Berry Républicain, 17 octobre 2013).

Lorsque l'on dispose de données nationales, une dernière question, autour du prononcé de la peine, est de savoir si certaines régions sont plus concernées par un phénomène ou si la juridiction qui a rendu la décision a eu un effet sur la peine prononcée. Pour l'ensemble des 357 cas découverts sur toute la période, seuls six départements atteignent ou dépassent la dizaine de cadavres: Bouchesdu-Rhône et Charente-Maritime (13 enfants), Ille-et-Vilaine (11), Gironde, Manche et Nord (10). Si I'on raisonne en nombre d'affaires et non en enfants, du fait des néonaticides multiples, seules les Bouches-du-Rhône et la Gironde dépassent la dizaine de cas. Des comparaisons fines nécessiteraient de calculer des taux par rapport au nombre de naissances de chaque département. Cela est peu susceptible de déboucher sur quelque chose de solide, compte tenu des très faibles effectifs. Les données sur les homicides en général par région montrent également des variations importantes (Mucchielli, 2009). Une fois de plus, on ne peut pas non plus exclure que certains journaux régionaux soient plus enclins à rapporter ces faits.

Peu de cas arrivent devant les différentes juridictions françaises. Les décisions dont nous disposons se dispersent sur 121 cours d'Assises. Pour 21 d'entre elles, un seul cas a été examiné en 20 ans. Le maximum est atteint par la Gironde qui a rendu six décisions.

\section{Les éléments liés aux acteurs}

Les affaires criminelles ici exposées sont évidemment examinées attentivement sur le fond, sur les éventuelles circonstances atténuantes, sur tous les éléments qui peuvent expliquer le geste meurtrier, donc les « raisons » de ce crime. Les explications de la mère sont un élément essentiel.

La mère essaye de se justifier et toutes les études historiques retiennent un certain nombre de constantes dans les explications fournies. Dans notre corpus, un recodage des explications avancées lors des procès permet de procéder à des regroupements en grandes catégories: le déni de grossesse (12\% des mères jugées), le refus d'un enfant supplémentaire (16\%), la peur de l'entourage ou du mari (13\%) associée ou non à une explication culturelle (12\%), la détresse ou la panique (18\%), les carences affectives ou l'enfance difficile (8\%), la mort résultant d'un accident 
jeudi 17 janvier 2019

pour lequel la mère clame son innocence $(3 \%)$, des relations sans lendemain ou adultérines (3\%). D'autres variables montrent un lien significatif avec ces sept catégories d'explications. Par exemple, quand plusieurs enfants ont été tués successivement, l'explication est plus souvent le refus d'un enfant supplémentaire. Pour les très jeunes mères, en particulier mineures, reviennent plus fréquemment la peur de l'entourage ou la panique totale. La peine ferme prononcée varie significativement avec ces explications: les peines les plus lourdes sont associées à un refus d'un enfant supplémentaire (plus de six ans ferme en moyenne), tandis que le déni ou l'explication culturelle (honte dans la communauté concernée) sont associés à des peines d'emprisonnement ferme de trois ans en moyenne, assorties de sursis partiel.

L'affaire Courjault a fait exploser la question du déni sur la scène médiatique, même si elle était déjà évoquée lors des procès. "Elle n'en parle à personne. La psychologue, Mme Hue-Pillette, explique ce "mécanisme bien connu du déni de grossesse" que l'on "rencontre fréquemment chez les adolescentes" ». (Sud-Ouest, 23 mars 2000). Aucune occurrence n'en apparaît dans la presse récoltée avant l'année 2000. II y a ensuite une accélération, au fur et à mesure de sa popularisation : 960 occurrences du mot déni peuvent être comptabilisées dans notre corpus. Depuis 2010, néanmoins le déclin de cette hypothèse est très net, y compris parce qu'elle est refusée fermement dans des cas très médiatiques, bien que ce soit un argument avancé aujourd'hui par tous les avocats des mères : " Joint au téléphone par l'AFP, Me Berton a également estimé que le procureur de la République de Douai, qui avait écarté a priori la thèse du déni de grossesse, était allé "un peu vite". (AFP, 20 juillet 2010). Ce même mouvement a été observé dans des études historiques à propos du recours à l'explication de la mère malade mentale pour expliquer le néonaticide, après des périodes où elle était considérée comme coupable puis d'autres où elle était décrite comme une victime (Tinkova, 2005). Ainsi, même s'il est difficile d'établir qu'il y ait un lien direct, la moyenne du quantum de la partie ferme de la peine prononcée par des arrêts de 1999 à 2004 ans était de 3,7 ans et a augmenté à 5,2 ans pour la période 2005-2010 puis à 6,6 ans après 2010 .

Le refus d'un enfant supplémentaire est l'explication la plus mal perçue par les jurys, tant les modes de vie actuels sont censés permettre de s'en protéger, l'IVG tout comme l'accouchement sous $X$ voire l'abandon sont disponibles. De ce fait, les femmes qui utilisent cet argument (dont toutes celles qui ont tué plusieurs enfants) sont souvent décrites comme froides ou au moins duelles pendant le procès et ne suscitent pas une particulière mansuétude. On trouve celles désignées comme bourreaux dans cette catégorie: "C'est à ce moment qu'elle aurait totalement perdu les pédales. "Tout d'un coup, ç'a été la peur, la panique, la colère, j'étais bonne à rien comme il me le répétait sans cesse. La haine est montée en moi.» Elle se déchaîne. Frappe le nouveau-né, toujours enveloppé dans son couvre-lit, contre le lavabo à plusieurs reprises. Au moins trois fois, diront les experts. Avec une violence inouïe ". (Charente-Libre, 16 janvier 2013). Cette explication, souvent combinée à une préméditation ou à l'absence de remords ( $c f$. infra), sera celle qui sera la moins susceptible d'atténuer la peine. "Elle aurait par ailleurs déclaré aux enquêteurs avoir abandonné ses enfants, car elle n'avait pas les "moyens de s'en occuper". Pour elle, les endroits où elle a déposé ses bébés étaient des lieux "visibles" afin qu'ils puissent être trouvés " (Le Parisien, 14 avril 2011).

La peur du mari ou de l'entourage est évoquée par des femmes qui sont décrites comme soumises à leur famille, sans grande autonomie. Elles ont souvent des rapports difficiles à leur corps ou aux médecins et ne se protègent pas des grossesses non désirées tout en vivant dans la crainte d'être enceinte et que cela déplaise à leur conjoint. "Quand elle devine sa grossesse, elle la refuse, la cache 
jeudi 17 janvier 2019

à la famille, à Bertrand. "Peur qu'il me quitte, lâche-t-elle les yeux baissés. Et si je lui disais, ma mère aurait été au courant.» Sarah retarde le moment de tout dévoiler. "Ma mère m'avait dit comme une menace: méfies-toi si tu tombes enceinte !» (Libération, 4 février 1995). Cette peur a été documentée dans un article précédent issu de l'analyse des dossiers judiciaires (Vellut, Cook and Tursz, 2012), mais aussi comme explication centrale d'une étude finlandaise (Amon et al., 2012).

La panique au moment de l'accouchement, suite à une grossesse cachée dont la mère a refusé d'imaginer le dénouement est fréquente. Les mères, plus souvent jeunes, sont totalement isolées et l'accouchement solitaire les amène à des actes de violence sur l'enfant "Paniquée" et "ne voyant pas d'autres solutions", elle avait tenté d'étouffer le nourrisson avec sa main, puis, croyant qu'il respirait toujours, elle l'avait poignardé à deux reprises avec un couteau de cuisine, selon les conclusions de l'enquête " (AFP, 28 mars 2010). N'oublions pas que les néonaticides font suite à des grossesses non déclarées, non ou très mal suivies médicalement.

La littérature sur les néonaticides, comme sur les meurtres d'enfants en général, retient généralement une dimension morale, les parents étant, par définition, les moins susceptibles d'avoir des excuses pour tuer leurs enfants. Néanmoins, dans le cas particulier du néonaticide, il est souvent admis qu'il s'agit d'un meurtre spécifique, non jugé en tant que tel à part entière, la mère étant susceptible d'être victime des circonstances, voire d'avoir le "droit ${ }^{12}$ " de refuser un enfant. Le défaut d'existence de l'enfant peut contribuer aux faibles peines, la personnalité de la mère, surtout si elle fait preuve d'empathie, prenant le pas sur celle de l'enfant.

Dans le récit qui est fait de l'audience pour une femme condamnée à quinze années d'emprisonnement ferme pour le meurtre d'un (seul) enfant, on retrouve les éléments déjà mis en exergue par l'analyse des dossiers judiciaires (Simmat-Durand, Vellut and Tursz, 2012) pour expliquer la lourdeur de cette peine : le mensonge, l'absence de compassion " Hier, la cour d'assises de l'Oise a condamné Nadine Bérube à quinze de réclusion criminelle pour avoir tué son bébé Maxime, le 10 février 2006 à Morvillers. Manipulatrice ou au contraire femme fragile ? ... Hier, ce sont surtout les mensonges de Nadine Bérube qui sont apparus. Et Martine Bouillon, l'avocate générale, s'est appuyée sur eux pour demander douze ans de réclusion criminelle. "Du début à la fin, elle a menti, clame-t-elle en montrant l'accusée du doigt. Elle a menti sur des détails, elle a menti aux experts, elle vous ment. On veut la présenter comme une femme fragile, ce n'est pas elle qui est fragile, c'est son bébé qui vient de naître. Plus il crie, plus elle est furieuse et plus elle tape. C'est ça, sa réponse. Son enfant n'est qu'une chose, elle ne dira jamais le mot bébé. " Et l'avocate générale de reprendre l'horrible scénario. "Elle n'est pas paniquée, elle se dépêche car elle sait que son compagnon va rentrer, détaille Martine Bouillon. Le bébé veut vivre, il pleure. Elle le met dans deux sacs-poubelle avant de le fracasser par trois fois avec une force extraordinaire selon l'expert. C'est de la barbarie ! (Le Parisien, 20 juin 2008).

Le fait pour la mère d'être capable de verbaliser, de convaincre les jurés de ses regrets, de sa honte, de ses remords pour ce crime joue sur la sanction, c'est en tout cas une piste que nous avions évoquée à partir des affaires judiciaires. Sur l'ensemble examiné ici, on retrouve cette même

\footnotetext{
${ }^{12}$ Selon le texte provocateur de deux philosophes, on devrait pouvoir tuer un nouveau-né comme on peut décider d'une IVG. "We claim that killing a newborn could be ethically permissible in all the circumstances where abortion would be," write Giubilini and Minerva. "Such circumstances include cases where the newborn has the potential to have an (at least) acceptable life, but the well-being of the family is at risk..." The argument by which they reach this position hinges on the idea that neither a fetus nor a newborn is a real person. (The Guardian, 29 February 2012).
} 
jeudi 17 janvier 2019

influence : de manière significative, la peine ferme varie de 8,76 ans en moyenne quand la mère n'a pas été capable de reconnaître l'enfant en tant que personne à 4,95 ans quand elle a exprimé des remords. "Tout en sanglotant, le corps penché en avant, les deux mains sur le rebord du box et le regard vers le sol, Stéphanie Parmentier s'est une dernière fois adressée à la cour : "Ma vie maintenant, tous les jours, c'est un cauchemar, même si je travaille, même avec ma famille, je revis tous les jours ce qui s'est passé, et ma fille, je suis allée la voir au cimetière, ça a été épouvantable. Au terme de deux longues journées d'audience, alors même que le procès n'était au départ prévu que sur un jour, la cour, présidée par M. Carbonell et les jurés, a en partie entendu la détresse de Stéphanie Parmentier en la condamnant à cinq ans de prison, dont deux avec sursis» (Sud-Ouest, 14 mai 1999).

\section{Une influence de la médiatisation?}

Le dévoilement de l'identité de la mère dans la presse, le fait de la présenter, de publier des interviews des proches, du voisinage pourrait avoir une influence sur l'opinion publique et donc sur les membres du jury, exposés eux aussi à cet environnement.

En théorie, ce type d'affaires est strictement protégé par le secret de l'instruction, les crimes et délits contre les enfants ne devant en particulier délivrer aucun élément d'identification. Le principe de présomption d'innocence voudrait que l'identité des protagonistes, et ici le plus souvent de la mère, ne soit divulguée qu'après la condamnation, puisque l'ouverture d'une enquête, voire d'une information judiciaire, ne présage pas de la culpabilité, un non-lieu ou un acquittement étant possible. Force est de constater que pour les affaires de meurtres d'enfants, ce secret de l'instruction n'est pas toujours respecté, le nom de la mère étant révélé de plus en plus tôt dans la procédure, le jour même de la découverte des cadavres bien souvent ou lors de la mise en examen. "La mère infanticide mise en examen. Blois. Mère de famille de 39 ans, Marinette Pezin a été mise en examen hier, à Blois, mais laissée en liberté sous contrôle judiciaire pour avoir tué trois de ses nouveau-nés dont les restes ont été découverts dans le jardin de son ancienne maison de Contres (Loir-et-Cher)" (Sud Ouest, 11 novembre 2006).

Dans l'ensemble des affaires complètes dont nous disposons, 53,2 \% mères ont été nominativement (nom et prénom) désignées dans la presse dont $18 \%$ avant le procès. De manière très significative, le nombre d'articles augmente quand l'identité est connue : la proximité est ainsi créée avec le lectorat. L'identité de la mère est divulguée le plus souvent le jour du procès, la session d'Assises étant publique, les journalistes y assistent. Pour les affaires antérieures aux années 2000, le nom n'est jamais cité avant l'audience, voire ne l'est jamais ${ }^{13}$. Après les années 2000 , une femme sur six a vu son identité figurer dans la presse dès la découverte, la mise en garde-à-vue ou la mise en examen.

L'affaire Cottrez est emblématique de cette tendance, puisque dès la découverte des cadavres, non seulement le pavillon a été photographié alors qu'il est situé dans une petite commune, le nom de la meurtrière présumée a été citée dans la presse régionale et nationale, une photographie d'elle a été publiée, reprise de sa page Facebook. Son visage devient celui de la plus grande infanticide française, dans le monde entier. Les nouveaux propriétaires du pavillon où les ossements ont été retrouvés

\footnotetext{
${ }^{13}$ Avant 2000, le quart des affaires examinées jusqu'au dénouement mentionne l'identité de la mère au procès, $5 \%$ la dévoile à la mise en examen ou avant.
} 
jeudi 17 janvier 2019

n'ont pas été épargnés et s'en sont fait l'écho au moment du procès : " Près de cinq ans que l'affaire a éclaté, et vous n'avez jamais parlé à la presse... L.M.: On avait peur d'être confrontés aux médias, peur que nos propos soient déformés. On a été assaillis par des dizaines de journalistes, des Anglais, des Italiens, les télés... Ils essayaient de filmer par-dessus le portail. Il y a même un avion qui a volé en rase-mottes pour prendre des photos du jardin. On voulait surtout protéger notre famille, nos enfants. J'ai appris quelques jours plus tard par une tante qui habite Annezin que j'étais en photo dans ParisMatch. On a attaqué, et gagné " (Nord Littoral, 6 mai 2015). C'est également une affaire pour laquelle des photographies pendant la session de la cour d'assises ont été publiées, les journalistes se contentant habituellement de croquis ou de photographies aux abords du Palais de Justice.

Pour d'autres, l'identité n'est pas révélée (elles ne sont pas comptées ci-dessus), mais tant de détails sont fournis dans la presse, que toutes ses relations, familiales, professionnelles ou de voisinage, peuvent l'identifier. "Un nouveau-né a été découvert mort dans une ferme du quartier des Beugnets. De nombreuses interrogations demeurent après la découverte, tôt hier matin, du corps sans vie d'un nouveau-né dans une ferme. Une ferme isolée, entourée de vignes et située sur les hauteurs du petit village de Triors. Une commune rurale aux 487 âmes se trouvant dans le Nord de la Drôme " (Le Dauphiné, 6 avril 2011).

Dans quelques articles, le souci de ne pas dévoiler l'identité de l'accusée est justifié "Le nom de l'accusé n'est pas indiqué pour protéger l'anonymat des victimes, comme le veut la loi » (Sud-Ouest, 13 octobre 2003). La protection des victimes, parties civiles ou de l'entourage nécessite la discrétion sur l'identité des personnes au moins pendant la phase de procédure avant jugement. Deux hypothèses non exclusives peuvent être avancées sur les évolutions en cours : les limites de la vie privée ont été repoussées par l'utilisation massive des réseaux sociaux et le corps du bébé, sans identité, n'est pas perçu comme une victime à protéger.

Certaines affaires semblent déchaîner la presse, permettant qu'un feuilleton s'installe, avec ses épisodes, ses détails et ses rebondissements, comme l'affaire Courjault en 2006 et l'affaire Cottrez ensuite. Cette " popularité " traduit soit l'horreur du crime, par exemple le record de nouveau-nés tués ${ }^{14}$, soit une fascination pour certaines circonstances, comme le fait d'avoir congelé le cadavre, soit des protagonistes célèbres, comme des avocats ou des experts réputés. Dans l'affaire Courjault, le fait que deux des cadavres aient été découverts à l'étranger, suscitant de premières et rocambolesques suspicions à l'égard du pays hôte a contribué à cet effet de fascination. Le calendrier n'est peut-être pas non plus une hypothèse à éliminer. L'affaire Courjault, comme l'affaire Cottrez, ont éclaté respectivement des 23 et 24 juillet. L'affaire de Cécile Lesage qui a pourtant tué six enfants a été découverte au mois d'octobre et ne s'est traduite que par 37 articles, dont six seulement lors de son procès, quelques mois après celui de Véronique Courjault, où elle a été condamnée à quinze ans de réclusion criminelle.

Le détail le plus rapporté par la presse est le lieu de la découverte du cadavre. Le nombre d'articles de presse varie considérablement en fonction de ce premier indice. Ainsi, les crimes les moins médiatisés sont ceux pour lesquels le cadavre a été découvert dans une maison ou son jardin, généralement moins de trois articles, mais avec l'exception notable de l'affaire Cottrez (mais c'est le

\footnotetext{
${ }^{14}$ Mais l'affaire de Céline Lesage qui a tué six nouveau-nés, citée ici, est passée relativement inaperçue lors de sa découverte, tout comme au moment de son procès, en décalage de quelques mois de celui de Véronique Courjault.
} 
jeudi 17 janvier 2019

jardin du nouveau propriétaire). Quand la découverte est faite dans un lieu public, l'intérêt du lectorat est éveillé par le recours à une forme de feuilleton : I'horreur de la découverte, la tenue en haleine des recherches, éventuellement une longue enquête appuyée par des prélèvements ADN, etc. Tous ces détails ressemblent à des émissions télévisées autour de séries criminelles. Les affaires entrant dans cette catégorie produisent le plus souvent de cinq à trente articles différents au moment de la découverte.

Enfin, une mention spéciale est réservée au congélateur. Si seulement $8 \%$ des nouveau-nés ont été découverts dans un congélateur (le plus souvent par le mari de la mère, son ex compagnon ou le père des enfants), un tiers des affaires a totalisé plus de trente articles. On voit s'exercer une fascination, relayée par des articles scientifiques proposant des éléments de théorie autour de la signification de ce geste. Ainsi, pour la psychiatre Sophie Marinopoulos, "Le dénominateur commun, c'est qu'elles ont gardé le ou les corps à proximité et qu'elles ont laissé des traces. Congeler le bébé, c'est préserver la vie à travers la mort. En même temps, le congélateur que tout le monde peut ouvrir est le dernier endroit sûr de la maison. Une part du sujet psychique de ces femmes a voulu qu'on puisse un jour les découvrir. Elles ont laissé quelque part la trace de leur souffrance ". (Sud-Ouest, 4 novembre 2006). Cette vision est confirmée par cette femme: " "Cet enfant je le voulais, assure-telle, je ne vis que pour les enfants. Le congélateur, c'était pour garder mon bébé près de moi, lance $t$ elle, et puis vous savez, si j'avais voulu m'en débarrasser, j'aurais pu l'enterrer et je ne serais pas là actuellement. Mais il fallait que je le garde" "(Sud-Ouest, 19 octobre 2000). D'autres mères concernées suggèrent plus prosaïquement que le congélateur se situe dans la cuisine, qui est leur territoire, ou qu'elles avaient peur de l'odeur, ou qu'elles avaient du mal à jeter leur enfant à la poubelle: "Je n'allais pas le jeter et je n'avais pas d'argent pour un enterrement" (Sud-Ouest, 8 décembre 2006). Au final, sur 154 mères jugées, 13 seulement avaient congelé le nouveau-né, essentiellement après son décès.

\section{Conclusion}

La forte médiatisation des affaires de néonaticides rendait intéressante la perspective d'utiliser la presse pour en savoir plus sur ces affaires rares et de ce fait dispersées sur nombre de juridictions françaises. La perspective prise ici, de collecter les coupures de presse sur vingt ans, offre une vue sur la situation nationale et permet de réaffirmer la remarquable stabilité de ce phénomène depuis la fin des années 1970 (Chesnais, 1976), entre une vingtaine et une trentaine de néonaticides découverts chaque année dans notre pays.

Tous les néonaticides ne sont sans doute pas rapportés dans la presse (c'est un crime relativement facile à cacher, sauf en cas d'hémorragie), mais globalement, ils le sont pour ceux qui sont découverts ou au moins pour ceux qui sont jugés, car ils font partie des faits divers attendus du lectorat des quotidiens régionaux. La mort violente d'un enfant, surtout très jeune, a donc une probabilité très élevée de report dans la presse. La comparaison avec l'étude antérieure portant sur des affaires identifiées à partir des tribunaux montre un bon taux de recouvrement au moins pour les affaires allant jusqu'au jugement, sauf quand l'auteur est mineur, car le procès peut se dérouler à huit-clos, mais nous disposons malgré tout du début de l'affaire avec le moment de sa découverte. 
jeudi 17 janvier 2019

L'intérêt évident de nos données est que nous disposons de plus de trois cents décès répartis sur une longue période sur tout le territoire national, alors que les données publiées comportent généralement de petites séries, soit parce qu'elles portent sur moins d'années, ou sur des régions, ou encore qu'elles sont obtenues à partir de cas cliniques. Nous disposons de plus, pour plus d'une centaine d'affaires poursuivies devant un tribunal, le plus souvent une cour d'assises, des décisions des cours et tribunaux, ce qui permet d'avoir une vision plus informée du traitement judiciaire. Les journalistes assistent généralement aux sessions d'assises et rapportent les débats. Plus l'affaire est complexe ou spectaculaire, plus elle est mise en scène et médiatisée, comme le montre le récent exemple du procès de Dominique Cottrez qui a généré plus de trois cents articles dans la seule presse française sur une semaine. Les dossiers judiciaires comportent quant à eux la totalité des procèsverbaux, expertises et analyses de la juridiction et sont donc bien plus riches et précis sur d'autres aspects.

La presse ne rapporte que rarement les décisions de classement sans suite des cas mentionnés. Quand l'affaire n'a pas été élucidée, l'absence d'auteur entraîne un abandon des poursuites et il n'y a aucun nouvel article. La durée moyenne des procédures judiciaires étant de plus de trois ans, les cas postérieurs à 2011 ne sont pas encore tous jugés. Pour les années antérieures, l'hypothèse que toutes les affaires qui ne sont plus mentionnées ultérieurement après la découverte ont été classées sans suite, soit $48 \%$, est cohérente avec d'autres données (Putkonen et al., 2007a). Dans notre étude, parmi 224 mères mises en cause, plus de la moitié ont été jugées. Toutes, sauf huit relaxées, ont été condamnées à une peine d'emprisonnement, dont les trois quarts à une peine comportant une partie d'emprisonnement ferme, 20 seulement ayant reçu un sursis probatoire. La situation française est donc assez éloignée de celle des pays comme le Royaume-Uni ou la Croatie qui préfère une peine probatoire, y compris parce que " another reason why mothers were rarely imprisoned is the belief that killing her own baby as produced enough of a sense of guilt to serve as punishment " (Marcikic et al. 2006). La question de savoir si ces femmes relèvent d'une sanction ou d'un suivi psychiatrique est posée dans de nombreux pays, comme au Canada (Laporte et al., 2003) ou en Finlande, où la faible utilisation des obligations de soins, y compris pour des femmes qui ont commis des néonaticides multiples ne manque d'interroger (Putkonen et al. 2007).

Le profil des mères est ce qui différencie le plus nettement les données françaises de celles publiées dans d'autres pays. Les données les plus courantes de la littérature sur les néonaticides font état de mères jeunes, isolées et de faible niveau éducatif, profil le plus courant dans les pays anglo-saxons où les grossesses précoces sont plus fréquentes (Friedman, Horwitz and Resnick, 2005). Plus récemment, dans une étude américaine de 45 cas, $68 \%$ des femmes étaient âgées de moins de 21 ans et parmi elles près de la moitié étaient lycéennes ou étudiantes (Shelton, Muirhead and Canning, 2010). Ce profil rejoint les descriptions historiques de la fille-mère, jeune, ne vivant pas en couple, dont la grossesse résulte de relations préconjugales voire adultérines (Tillier, 2001). Quelques études montrent que le profil des mères en France (Vellut, Simmat-Durand and Tursz, 2013) ou par exemple en Croatie (Marcikic et al., 2006) est beaucoup plus diversifié, ce que nous observons ici avec des femmes plutôt multipares, d'âge habituel à la maternité. Néanmoins, la proportion de femmes âgées de moins de 25 ans est conséquente, $38 \%$, alors que cette classe d'âges n'est à l'origine que de $16 \%$ des naissances en France. 
jeudi 17 janvier 2019

Cinq autres résultats intéressants ont émergé de cette analyse, permis par le nombre important d'affaires, dont certains seulement avaient été ébauchés à partir de l'analyse des dossiers judiciaires (Simmat-Durand, Vellut and Tursz, 2012).

Le premier est qu'en filigrane de toute la prise en charge judiciaire de ces affaires, les circonstances de la découverte ont une influence déterminante qui persiste tout au long de la procédure. Pour les femmes les plus âgées, déjà mères de famille, qui sont les plus nombreuses dans les données françaises, la probabilité de découverte immédiate est relative, sauf si ces mères nécessitent une hospitalisation du fait d'une hémorragie : les cadavres sont bien dissimulés, au plus près ou dans leur lieu d'habitation. Ceux qui sont découverts, le sont finalement à l'occasion d'un déménagement, d'un changement de vie ou de conjoint, ou à la suite d'une dénonciation au sein du couple, de la famille ou du voisinage, quand un conflit apparaît. La découverte peut ainsi n'avoir lieu qu'après la période de prescription, bien que la position de la Cour de Cassation lors de l'affaire Cottrez ait abrogé cette notion, les poursuites ayant été considérées comme possibles en faisant courir le délai de prescription de la découverte des cadavres et non du meurtre.

Le second est que l'on assiste pour ces affaires fortement médiatisées sur la période à une " fin » de l'anonymat et de la présomption d'innocence : ces femmes sont nommément visées de plus en plus tôt après la découverte du cadavre, ou des photos permettent de savoir au moins localement de qui il s'agit (pavillon dans un tout petit village...). Les affaires les plus anciennes ne révélaient pas le nom de la mère, du moins pas avant le procès en cour d'assises et la condamnation. Dans les années de début de période de recueil, même le prénom du bébé décédé était modifié dans la presse, l'article en portait I'indication. On assiste de ce fait pour ces femmes à une évolution qui conduit à un lynchage médiatique relevant tout à la fois de la sanction morale contre la mère et de la non reconnaissance du statut de victime mineure pour l'enfant dont l'existence n'est pas bien affirmée. Ce point méritera une étude ultérieure, prenant en compte des données encore plus récentes.

Une constante historique pour la France, en troisième lieu, qui est la non mise en cause des pères dans ces affaires, sauf à de très rares exceptions près, comme si l'enfant n'avait pas été conçu au sein du couple et quand bien même le père aurait menacé la mère sur la question d'un enfant supplémentaire. Le néonaticide reste un crime féminin, solitaire, une " histoire tue " (Vellut, SimmatDurand and Tursz, 2015) et l'action judiciaire maintient ce dimorphisme sexuel depuis des siècles.

Au fil des années également, émerge le quatrième résultat qui est le développement, puis la quasi disparition, de l'explication par le déni de grossesse, tendant à montrer que la mère n'est ni criminelle, ni victime, renouant avec le mouvement observé à la fin du $19^{\text {ème }}$ siècle d'une psychopathologisation: "la grossesse, les règles, l'accouchement, la lactation étaient présentés comme des états anormaux, pathologiques, entraînant souvent des aliénations de courte durée... qui contribua à atténuer la responsabilité des mères " (Tinkova, 2005). Cette explication pathologique par le déni semble s'amplifier autour de l'affaire Courjeault, pour ensuite diminuer de nouveau, la notion de grossesse cachée prenant le dessus, car souvent la mère a reconnu qu'elle se savait enceinte, mais qu'elle a voulu l'ignorer.

Enfin le cinquième et dernier résultat que nous voulons mettre en valeur ici est l'influence sur la peine des explications fournies par la mère, son passé, sa personnalité, son vécu, sa capacité à verbaliser, à exprimer des remords ou à faire enfin vivre cet enfant en le reconnaissant devant le jury. Le cas très particulier des infanticides multiples sera repris ultérieurement, tant ces actes 
jeudi 17 janvier 2019

répétitifs semblent mettre à mal les explications habituellement retenues pour un acte unique. L'interaction au moment du procès entre le passé de cette mère, son apparence, son attitude, ses explications et le jury est un apport important de ce qui figure dans la presse, les dossiers judiciaires ne comportant que peu de notes sur les débats, le jugement en lui-même ne retenant que ce sur quoi est basée la décision.

De nombreuses thématiques restent à approfondir à partir de ces données par une analyse de contenu encore plus fine, de la mise en scène du récit, du rôle du père qui de non mis en cause devient partie civile, des divers éléments influençant la décision judiciaire et surtout la variabilité du quantum de la peine, voire des représentations autour de la mère néonaticide véhiculées par la presse sur les vingt dernières années. Une première piste intuitive à la lecture de tout ce matériau, est d'expliquer comment au fil de cette médiatisation d'affaires dont les protagonistes sont connus du grand public, voire "vedettes" de reconstitutions ou d'émissions, la mère néonaticide a pu devenir sympathique dans la presse française, alors qu'elle était présentée comme un monstre en début de période.

Remerciements :

Anne Toppani, Ingénieure au Cermes3 pour sa participation à la première phase du recueil des données. 
jeudi 17 janvier 2019

Figure 1 : Corpus, nombre de cas élucidés et non élucidés par année et taux pour 100000 naissances

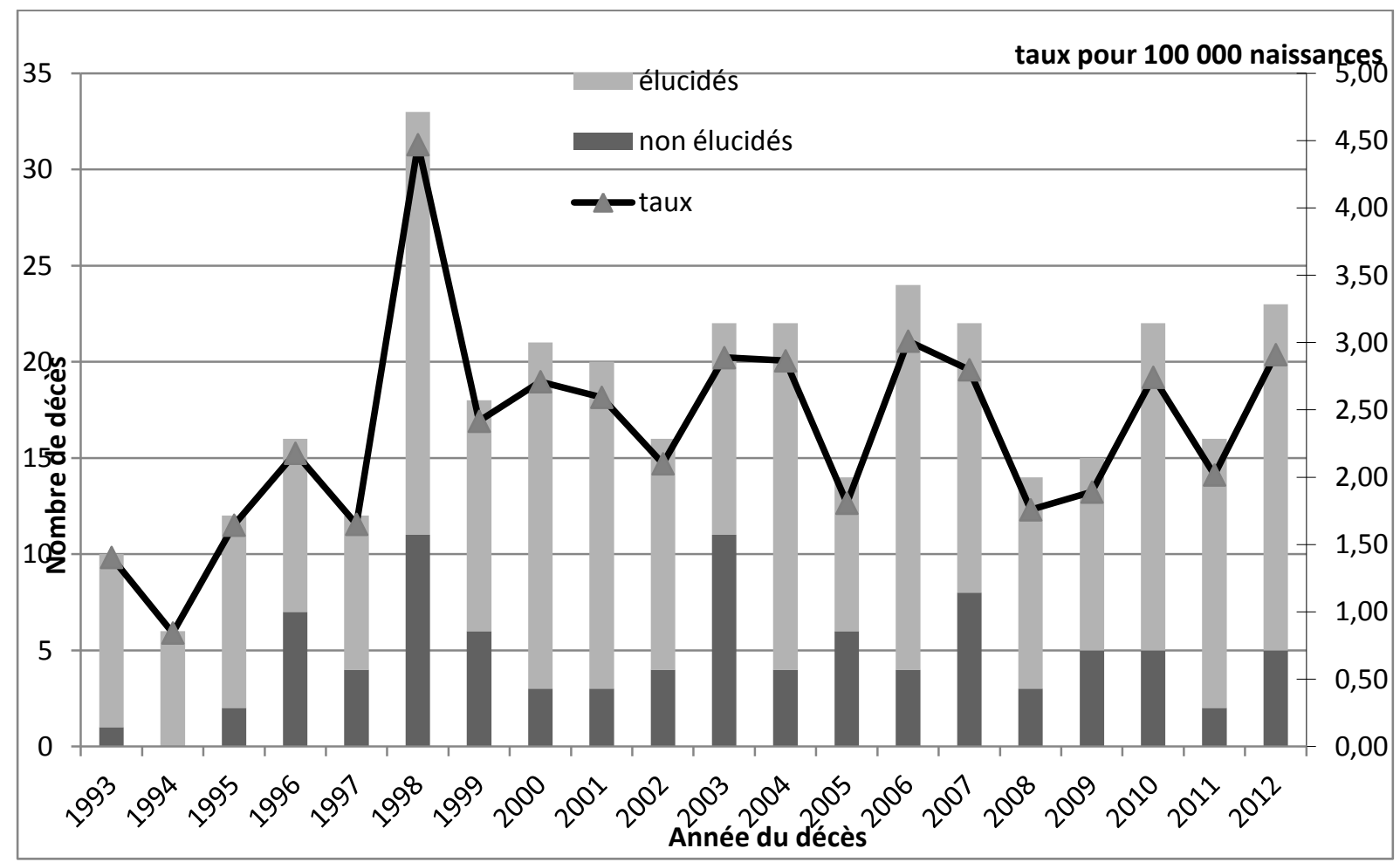


jeudi 17 janvier 2019

Figure 2 : France, Faits constatés par les services de police et de gendarmerie et condamnations portées au casier judiciaire, pour des faits d'infanticides ou de meurtres sur victimes de moins de quinze ans

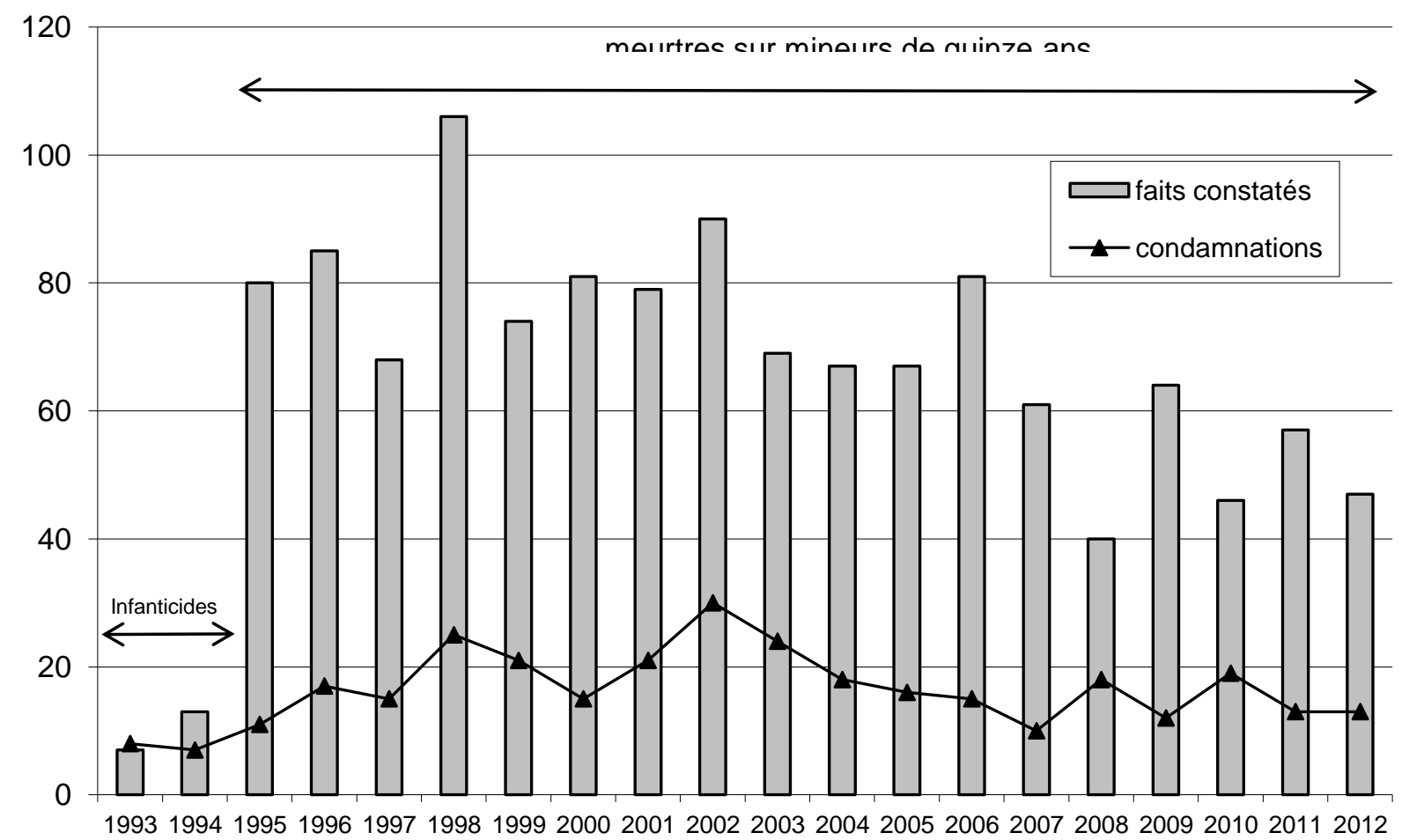


jeudi 17 janvier 2019

Références

AMON S., PUTKONEN H., WEIZMANN-HENELIUS G., ALMIRON M. P., FORMANN A. K., VORACEK M., ERONEN M., YOURSTONE J., FRIEDRICH M., and JKLIER C. M., 2012, "Potential predictors in neonaticide: the impact of the circumstances of pregnancy," Archives of Women's Mental Health 15:167-74.

BYDLOWSKI M., 2011, "Le néonaticide ou infanticide néonatal," Perspectives Psychiatriques 50(4):311-13.

CHESNAIS J.-C., 1976, Les morts violentes en France depuis 1826, Paris: Presses Universitaires de France.

CRAIG M., 2004, "Perinatal risk factors for neonaticide and infant homicide: can we identify those at risk?," Journal of the royal society of medecine 97(2):57-61.

DEAN P. J., 2004, "Child homicide and Infanticide in New Zealand," International Journal of Law and Psychiatry 27(4):339-48.

DUBÉ M., LÉVEILlÉE S., and MARLEAU J. D., 2003, "Cinq cas de néonaticide au Québec," Sante mentale au Quebec XXVIII(2):183-94.

FRIEDMAN S. H., HORWITZ S. M., and RESNICK P. J., 2005, "Child Murder by Mothers: A Critical Analysis of the Current State of Knowledge and a Research Agenda," American Journal of Psychiatry Vol 162(9):1578-87.

GEOFFROY-POISSON S., 2005, "L'infanticide devant la cour d'assises de la Haute-Marne au XIXè siècle," Les Cahiers du Centre de Recherches Historiques 35.

HENRY A., 2009, "Suivis de femmes infanticides à la prison de Rennes," Enfances \& Psy 44:84-91.

HERMAN-GIDDENS M. E., SMITH J. B., MITTAL M., CARLSON M., and BUTTS J. D., 2003, "Newborns Killed or Left to Die by a Parent " The Journal of The American Medical Association 289(11):1425-29.

KRÜGER P., 2015, "Prevalence and phenomenology of Neonaticide in Switzeland 1980-2010: A restrospective study," Violence and Victims 30(2):194-207.

LAPORTE L., POULIN B., MARLEAU J., ROY R., and WEBANCK T., 2003, "Filicidal women: Jail or psychiatric ward?," Canadian journal of psychiatry 48(2):94-98.

LÉAUTÉ J., 1968, Recherches sur l'infanticide (1955-1965), Paris: Dalloz.

MARCIKIC M., DUMENCIC B., MATUZALEM E., MARJANOVIC K., POZGAIN I., and UGLAREVIC M., 2006, "Infanticide in Eastern Croatia," Coll Antropol 30(2):437-42.

MENDLOWICZ M. V., RAPAPORT M. H., MECLER K., GOLSHAN S., and MORAES T. M., 1998, "A CaseControl Study on the Socio-Demographic Characteristics of 53 Neonaticidal Mothers " International Journal of Law and Psychiatry 21(2):209-19.

MUCCHIELLI L., 2009, "Les homicides dans la France contemporaine (1970-2007) : évolution, géographie et protagonistes," Pp. 133-64 in Histoire de l'homicide en Europe, edited by Laurent Mucchielli and Pieter Spierenburg. Paris: La découverte.

OTTESEN V., 2012, "A current absence of neonaticide in Norway," Forensic science 18(2):155-63.

PUTKONEN H., COLLANDER J., WEIZMANN-HENELIUS G., and ERONEN M., 2007a, "Legal outcomes of all suspected neonaticides in Finland 1980-2000 " International Journal of Law and Psychiatry 30(3):248-54.

PUTKONEN H., WEIZMANN-HENELIUS G., COLLANDER J., SANTTILA P., and ERONEN M., 2007b, "Neonaticides may be more preventable and heterogeneous than previously thought-Neonaticides in Finland 1980-2000," Archives of Women's Mental Health 10(1):15-23.

RAPAPORT E., 2006, "Mad women and desperate girls: Infanticide and child murder in Law and Myth," Fordham Urb. L.J. 33:527-69.

RESNICK P. J., 2009, "Retour sur le néonaticide : perspectives actuelles," Enfances \& Psy 44(3):55-59. 
jeudi 17 janvier 2019

SHELTON J., COREY T., DONALDSON W., and DENNISON E., 2011, "Neonaticide: A Comprehensive Review of Investigative and Pathologic Aspects of 55 Cases," Journal of family violence 26(4):263-76.

SHELTON J. L. E., MUIRHEAD Y., and CANNING K. E., 2010, "Ambivalence toward mothers who kill: An examination of 45 U.S. cases of maternal neonaticide," Behavioral Sciences \& the Law 28(6):812-31.

SIMMAT-DURAND L., and VELLUT N., 2013, "Le corps sans existence : les enfants victimes de néonaticides," Corps Quelle conscience de son corps ?(11):243-53.

SIMMAT-DURAND L., VELLUT N., and TURSZ A., 2012, "Les néonaticides devant la justice : le reflet d'une ambivalence face à ces crimes ?," Déviance et Société 36(4):413-40.

TILLIER A., 2001, Des criminelles au village. Femmes infanticides en Bretagne (1825-1865), Rennes: Presses Universitaires de Rennes.

TINKOVA D., 2005, "Protéger ou punir ? Les voies de la décriminalisation de l'infanticide en France et dans le domaine des Habsbourg (XVIIIè-XIXè siècles)," Crime, Histoire \& Sociétés 9(2):43-72.

TURSZ A., and COOK J. M., 2011, "A population-based survey of neonaticides using judicial data," Archives of Disease in Childhood. Fetal and Neonatal Edition 96:F259-F63.

TURSZ A., CROST M., GERBOUIN-RÉROLLE P., and COOK J. M., 2010, "Underascertainment of child abuse fatalities in France: Retrospective analysis of judicial data to assess underreporting of infant homicides in mortality statistics," Child Abuse \& Neglect 34(7):534-44.

TURSZ A. D., SIMMAT-DURAND L., GERBOUIN-RÉROLLE P., VELLUT N., and COOK J., 2011, "Les morts violentes de nourrissons : trajectoires des auteurs, traitement judiciaire des affaires," Pp. 118. Paris: ONED.

VELLUT N., COOK J. M., and TURSZ A., 2012, "Analysis of the relationship between neonaticide and denial of pregnancy using data from judicial files," Child Abuse \& Neglect 36(7-8):553-63.

VELLUT N., and SIMMAT-DURAND L., 2013, "L'influence des expertises psychiatriques sur la décision judiciaire : le cas des néonaticides," La Revue de Médecine Légale 4:75-83.

VELLUT N., SIMMAT-DURAND L., and TURSZ A., 2013, "Le portrait des mères néonaticides dans les expertises judiciaires," L'Encéphale 39:352-59.

-, 2015, "Accoucher sans donner naissance : les néonaticides, des histoires tues," Recherches Familiales 12(Numéro spécial Naître):99-112.

YASUMI K., and LAGEYAMA J., 2009, "Filicide and fatal abuse in Japan, 1994-2005: Temporal trends and regional distribution," Journal of Forensic and Legal Medicine 16:70-75. 